\title{
The effects of acupuncture on pregnancy outcomes of in vitro fertilization: a systematic review and meta-analysis
}

Zheng-yun Xie ${ }^{1}$, Zhi-hang Peng ${ }^{2}$, Bing Yao ${ }^{3}$, Li Chen ${ }^{3}$, Yan-yun Mu${ }^{4}$, Jie Cheng ${ }^{4}$, Qian Li ${ }^{4}$, Xi Luo ${ }^{5}$, Peng-yan Yang ${ }^{6}$ and You-bing $\mathrm{Xia}^{7^{*}}$ (D)

\begin{abstract}
Background: The effects of acupuncture on in vitro fertilization (IVF) outcomes remain controversial. And the variation in participant, interventions, outcomes studied, and trial design may relate to the efficacy of adjuvant acupuncture.

Methods: We searched digital databases for relevant studies, including Embase, PubMed, Cochrane Library and some Chinese databases up to December 2018, for randomized controlled trials (RCTs) evaluating the effects of acupuncture on women undergoing IVF. We included studies with intervention groups using needling, and control groups consisting of no acupuncture or sham (placebo) acupuncture. Primary outcomes were clinical pregnancy rate (CPR) and live birth rate (LBR). Meta-regression and subgroup analysis were conducted on the basis of eight pre-specified covariates to investigate the variances of the effects of adjuvant acupuncture on pregnancy rates and the sources of heterogeneity.
\end{abstract}

Results: Twenty-seven studies with 6116 participants were included. The pooled clinical pregnancy rate (CPR) from all of acupuncture groups was significantly greater than that of control groups (RR 1.21, 95\% Cl: 1.07-1.38), whereas the pooled live birth rate (LBR) was not. Meta-regression subgroup analysis showed a more significant benefit of acupuncture for repeated IVF cycle proportion (number of women with a history of prior unsuccessful IVF attempt divided by number of women included in each trial) $\geq 50 \%$ group (CPR: RR 1.60, 95\% Cl: 1.28-2.00; LBR: RR 1.42, 95\% Cl: 1.05-1.92), and this covariate explained most of the heterogeneity (CPR and LBR: adjusted $R^{2}=100$ and 87.90\%). Similar results were found between CPR and number of acupuncture treatments (CPR: $p=0.002$, adjusted $R^{2}=51.90 \%$ ), but not LBR.

Conclusions: Our analysis finds a benefit of acupuncture for IVF outcomes in women with a history of unsuccessful IVF attempt, and number of acupuncture treatments is a potential influential factor. Given the poor reporting and methodological flaws of existing studies, studies with larger scales and better methodologies are needed to verify these findings.

Keywords: Acupuncture, In vitro fertilization, Clinical pregnancy rate, Live birth rate, Systematic review

\footnotetext{
* Correspondence: youbingxia@hotmail.com

${ }^{7}$ Xuzhou Medical University, Xuzhou, China

Full list of author information is available at the end of the article
}

(c) The Author(s). 2019 Open Access This article is distributed under the terms of the Creative Commons Attribution 4.0 International License (http://creativecommons.org/licenses/by/4.0/) which permits unrestricted use, distribution, and reproduction in any medium, provided you give appropriate credit to the original author(s) and the source, provide a link to the Creative Commons license, and indicate if changes were made. The Creative Commons Public Domain Dedication waiver (http://creativecommons.org/publicdomain/zero/1.0/) applies to the data made available in this article, unless otherwise stated. 


\section{Introduction}

$12.5 \%$ women failure to establish a clinical pregnancy after 12 months of regular, unprotected sexual intercourse $[1,2]$. One of the most successful and commonly utilized treatment options is IVF and its related derivative technologies. In 2011 worldwide, approximately 2.0 million IVF cycles were reported, resulting in 0.5 million babies born [3]. Over the past few decades, technological advances in IVF, including advances in protocols for ovarian stimulation, oocyte retrieval, fertilization, and embryo culture and transfer, have resulted in more efficient approaches to treating infertility [4]. Nevertheless, delivery rates (DR) per aspiration remain low, with rates of $19.8 \%$ in 2011 worldwide [3], and $22.3 \%$ in Europe, 2013 [5]. For many women, cycles need to be repeated to be successful. Moreover, the IVF cycle is time-consuming and costly, including controlled ovarian hyperstimulation $(\mathrm{COH})$, oocyte retrieval, IVF/intracytoplasmic sperm injection (ICSI), embryo transfer (ET) and luteal support, with costs range from $\$ 15,000$ to $\$ 18,000$ per cycle, including medications. And the preimplantation genetic diagnosis (PGD) step of the process meant another $\$ 3000$ to $\$ 6000$. Altogether, conservatively speaking, about $\$ 20,000$ [6-9]. Methods that are effective in improving IVF outcomes may reduce the need for an additional high-cost IVF cycle. Therefore, there is a need to explore new techniques and therapies to improve the success rates of IVF.

Acupuncture and other modalities of Chinese/East Asian medicine have been used in women's health for many centuries. They are safe in the hands of competent practitioners $[10,11]$, even for pregnant women suffering various complications (e.g. hyperemesis gravidarum) $[12,13]$. Many studies have reported that acupuncture improved pregnancy rates among women undergoing IVF [14-22], which may relate to the following mechanisms, including regulating the function of the hypothalamic-pituitary-ovarian axis by changing the concentration of central opioids [23-26], improving blood circulation to the uterus and ovaries by inhibiting uterine central sympathetic nerve activity $[17,27]$ and reducing stress, anxiety or depression [28-30]. Subsequently, many systematic reviews analyzed the effects of acupuncture among women undergoing IVF [31-43], however, these meta-analyses have led to different conclusions. Potential reasons for these discrepancies include differences in how studies are selected, and how participants, interventions and outcomes are defined by reviewers [44]. Consequently, it may be difficult to draw definitive conclusions based on previous meta-analyses. Hence, we conducted a new systematic review and meta-analysis of randomized controlled trials (RCTs) involving previously defined set of subgroup analyses and meta-regressions to explore whether variation among participants, interventions, outcomes studied, and trial design influence estimates of the effects of adjuvant acupuncture on pregnancy outcomes in women undergoing IVF.

\section{Methods}

\section{Search strategy}

We searched digital databases for relevant studies, including Embase, PubMed, Cochrane Library and some Chinese databases, such as WanFang, CNKI, VIP and the Chinese SinoMed Database (up to December 2018). The MEDLINE search strategy is given in Additional file 4: Table S1.

The following were used as free text terms and $\mathrm{MeSH}$ terms: acupuncture therapy; acupuncture; electroacupuncture; auriculotherapy; auricular acupuncture; acupuncture analgesia; acup* and reproductive techniques, assisted; assisted reproducti*; in vitro fertili*; intracytoplasmic sperm injection; embryo transfer; embryo implantation; oocyte; egg collection. We combined this search strategy with a filter for clinical trials.

The following terms were used in the Chinese database searches: "ZHEN JIU" (which means "acupuncture and moxibustion"); "ZHEN CI" (which means "acupuncture"); "DIAN ZHEN" (which means "electroacupuncture"); "ER ZHEN" (which means "auricular acupuncture") and "FU ZHU SHEN ZHI" (which means "assisted reproductive technology"); “TI WAI SHOU JING” (which means "in vitro fertilization"); "SHI GUAN YING ER” (which means "test tube baby"); "LUAN BAO JIANG NEI DAN JING ZI XIAN WEI ZHU SHE" (which means "intracytoplasmic sperm injection"); PEI TAI YI ZHI (which means "embryo transfer").

We also scanned the Index to Scientific \& Technical Proceedings (Web of Science), and the reference lists of relevant primary and review articles were examined to identify cited articles not captured by electronic searches.

\section{Study selection}

The inclusion criteria were as follows: (1) RCTs that evaluated the effects of acupuncture on IVF outcomes in women undergoing IVF, with or without ICSI. Namely, women in intervention groups received both IVF and acupuncture and control groups received IVF with or without sham/placebo acupuncture; (2) no restriction on objective of study, meaning we included studies where acupuncture was administered for pain relief during oocyte retrieval, or for anxiety relief during IVF-ET, or for improving IVF outcomes; (3) any of three types of acupuncture: manual (MA), electrical (EA), and auricular acupuncture techniques; (4) studies using either traditional acupuncture, in which needles were inserted in classical meridian points, or western medical acupuncture [45], in which the needles were inserted in non- 
meridian or trigger points; (5)a clear description of acupuncture time. That is, we included studies which give an equal number of acupuncture treatments for women within a group; (6) needling in the control groups could be either no acupuncture or sham (placebo) acupuncture; (7) both fresh and frozen-thawed embryo transfer cycles reporting at least one of the following outcomes: clinical pregnancy rates $(\mathrm{CPR}$ - a pregnancy diagnosed by ultrasonographic visualization of one or more gestational sacs or definitive clinical signs of pregnancy), or live birth rates (LBR - the complete expulsion or extraction from a woman of a product of fertilization, after 22 completed weeks of gestational age; which, after such separation, breathes or shows any other evidence of life, such as heart beat, umbilical cord pulsation or definite movement of voluntary muscles, irrespective of whether the umbilical cord has been cut or the placenta is attached) [2]; and (8) no restrictions on publication type or language. Where studies had multiple publications, the main trial report was used as the reference and additional details were derived from secondary papers.

Exclusion criteria were as follows: studies of acupuncture treatments without needling; retrospective studies, case series, and studies with a crossover design.

\section{Data extraction}

Specific characteristics were extracted from each study: first author, year, demographic characteristics (i.e. age, IVF cycle number, duration of infertility, type of infertility, number of embryos transferred), invention (i.e. acupuncture type, acupoints, acupuncture time, number of acupuncture treatments), type of control, IVF outcomes and methodological quality of the trials.

Study selection and data extraction were completed in duplicate and independently by two investigators (ZYX and XL). A third reviewer (ZHP) independently assessed the study for consensus in case of disagreement. We corresponded with study investigators to clarify further data on methods and results. The relevant primary and review articles were also examined to identify further data on methods and results not published in the papers.

\section{Quality assessment}

Included studies were assessed for risk of bias using the Cochrane risk of bias assessment tool [46] to assess the following domains: random sequence generation; allocation concealment; blinding of patients; incomplete outcome data; selective reporting; and other bias. The GRADE methodology was used to assess the quality of retrieved evidence (GRADEpro, Version 3.6 for Windows, Grade Working group). The level of evidence was categorized into 4 levels: high, moderate, low, or very low quality.

\section{Outcome measures}

We pre-specified clinical pregnancy rate $(\mathrm{CPR})$ and live birth rate (LBR) as our primary outcomes.

Miscarriage rate (MR: [CPR-LBR]/CPR) and any reported side effects from the treatments were analyzed as secondary outcomes.

\section{Statistical analysis}

Data were analyzed in accordance with the Cochrane Handbook for Systematic Reviews of Interventions [47]. All data were dichotomous. Results were pooled and expressed as relative risks (RR) with $95 \%$ confidence intervals (CI) using RevMan V.5.3 meta-analysis software (The Nordic Cochrane Centre, The Cochrane Collaboration, 2014). Because of the expected heterogeneity of acupuncture protocols, controls, and demographic characteristics, a random effects model was used. Heterogeneity of treatment effects was evaluated graphically using Forest plot, and statistically by $I^{2}$ statistic and chi-square test. We performed both an available case analysis for a main analysis and an intention-to-treat analysis (ITT) for sensitivity analysis, which means all of the metaanalyses were based on both the number of whose results are known (available case analysis) and the number of women randomized (ITT).

\section{Subgroup analyses}

We conducted subgroup analyses on the basis of the following covariates: (1) age (<33.3 or $\geq 33.3$ years); (2) duration of infertility $(<5.6$ or $\geq 5.6$ years). Mean age was 33.3 years and duration of infertility was 5.6 years for the largest IVF success rates prediction study $(144,018$ cycles) which assessed the extent to which baseline characteristics be predict pregnancy rates [48]; (3) type of infertility (primary infertility proportion $<50 \%$ or $\geq 50 \%$ ); (4) repeated IVF cycle proportion (number of women with a history of prior unsuccessful IVF attempt divided by number of women included in each trial) $<50 \%$ or $\geq$ $50 \%$; (5) number of embryos transferred $(<1.9$ or $\geq 1.9$, which was the average number of embryos transferred in 2011 globally [3]); (6) number of acupuncture treatments (one or more than one session). We classified protocols in which acupuncture was performed $25 \mathrm{~min}$ prior to and after ET as one session, others counted as descriptions in each study. This is because embryo transfer requires only a few minutes, and the interval of two acupuncture treatments performed $25 \mathrm{~min}$ before and after ET is very short. Considering that the physiological effects of acupuncture can continue for many hours [49,50], it is reasonable to classify this protocol as one acupuncture treatment; (7) acupuncture type (electroacupuncture or 
manual acupuncture); (8) type of control (no or sham (placebo) acupuncture invention control).

Subsequently, a random effects meta-regression analysis was conducted for each variable in STATA version 14.0 (Stata Corporation, College Station, TX, USA). $P$-value was significant at the 0.05 level. Each variable tested independently in the model. Considering meta-analysis allows for residual heterogeneity among intervention effects not modelled by the explanatory variables, it is reasonable to use a random effects meta-regression analysis [51]. Due to some variables in our meta-analysis being mean values, we also considered the existence of aggregation bias (also known as ecological bias or the ecological fallacy) [52-54]. Multivariate meta-regression was not conducted in our meta-analysis primarily because of the limited number of included studies for each subgroup and our objective was to identify the relevant influential factors for the effects of acupuncture on IVF outcomes.

\section{Publication bias}

Publication bias was visually evaluated using funnel plots when at least 10 trials were available for meta-analysis. Subsequently, Egger's test [55] was performed to statistically assess the degree of asymmetry, and a cumulative meta-analysis was conducted to identify the potential for small-study effects. A two-tailed $p$ value $<0.05$ was considered statistically significant.

\section{Sensitivity analyses}

The sensitivity analyses were performed to explore whether the overall conclusions were affected under the four scenarios: (1) if we included randomized participants whose pregnancy outcome data were missing; (2) if we re-classified before and after ET protocols into two acupuncture sessions; (3) if we restricted CPR results to the 15 studies which reported LBR; and (4) if we performed the meta-regression and subgroup analyses stratified by control type.

In addition, we performed the meta-regression subgroup analyses according to 'risk of bias' of included studies.

\section{Results}

The selection process is documented in a PRISMA flow chart in Additional file 1: Figure S1. Reasons for excluding studies are given in Additional file 8: Appendix 1. Twenty-seven randomized controlled trials [14-22, 29, 56-72] with a total of 6116 participants met inclusion criteria. The characteristics of the included studies are displayed in Table 1.

\section{Inclusion criteria}

The differences among inclusion criteria for 27 studies are as follows:
Two German trials $[14,71]$ included only women with good-quality embryos, and one trial [21] restricted eligibility to women who had at least two previous unsuccessful attempts of IVF. The other trials included women with varying quality of embryos and number of previous IVF attempts. One trial [18] restricted eligibility to women with unfavorable reproductive prognoses, and others included women without limit to reproductive prognoses or ovarian responses. One trial [60] included participants who were infertile with polycystic ovary syndrome (PCOS) and candidates for IVF/ICSI, others included women with various causes of infertility. One trial [63] restricted eligibility to frozen-thawed embryo transfer cycles, and one [22] included both frozenthawed and fresh embryos, while the others used fresh embryos. One trial [64] used ICSI for all participants, whereas others used of ICSI for only some participants.

\section{Acupuncture interventions}

The timing of the acupuncture sessions differed somewhat among trials. Four studies [68-70, 72] performed one acupuncture session during oocyte aspiration (OA). Eleven studies [14, 19, 29, 57, 59, 61, 63-71] performed one or two acupuncture sessions around embryo transfer. Twelve studies performed two or more than two acupuncture sessions. Among these, ten [17, 18, 20-22, $56,58,60,62,67]$ performed during $\mathrm{COH}$ with or without ET day, two $[15,16]$ performed around and 2-3 days after ET.

Seven studies [17, 22, 60, 68-70, 72] used EA, and the other 21 trials used manual acupuncture (MA) as adjunctive treatment. Six trials [19, 22, 29, 57, 68, 69] did not report whether the needles used in the true acupuncture group were manipulated to achieve the de qi sensation (i.e. a sensation perceived by the patients, which manifests as numbness, heaviness, distention, and soreness, with spreading sensation and it is also perceived by the acupuncturists, which manifests as heavy and tight sensation coming from beneath the needle [73].) The remaining 21 trials needles were manually stimulated to obtain de qi sensation. Both Sator-Katzenschlager et al. [68] and Westergaard et al. [16] had two intervention groups, the former using auricular electro-acupuncture and auricular acupuncture, and the latter performed MA in different times between two invention groups. One trial [59] used off-site acupuncture, which means only patients in the acupuncture group were required to drive to and from the off-site acupuncturist's office both before and after the embryo transfer procedure, one [56] adopted both on-site and off-site acupuncture, and the remaining 25 trials were all on-site acupuncture. 


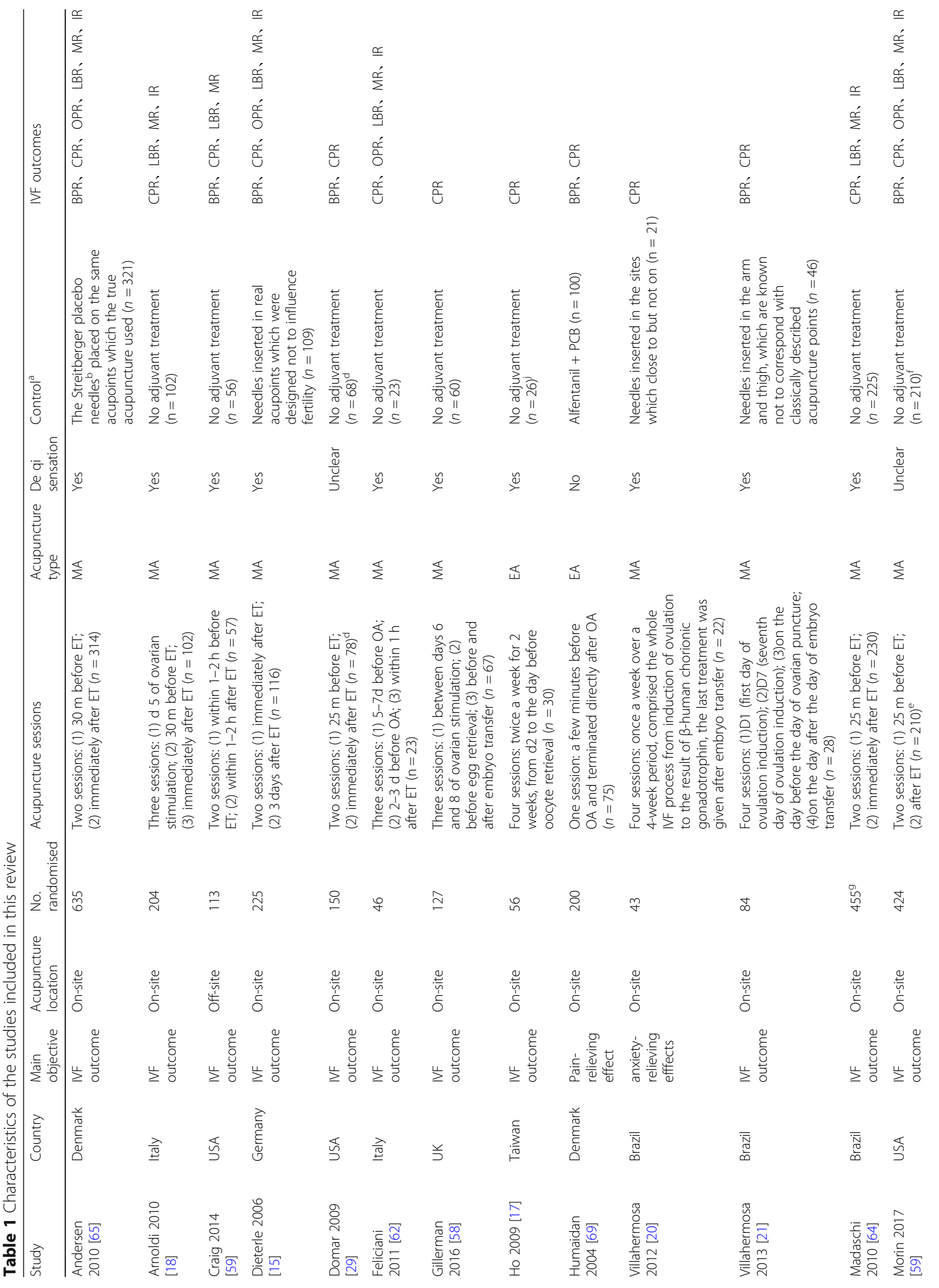




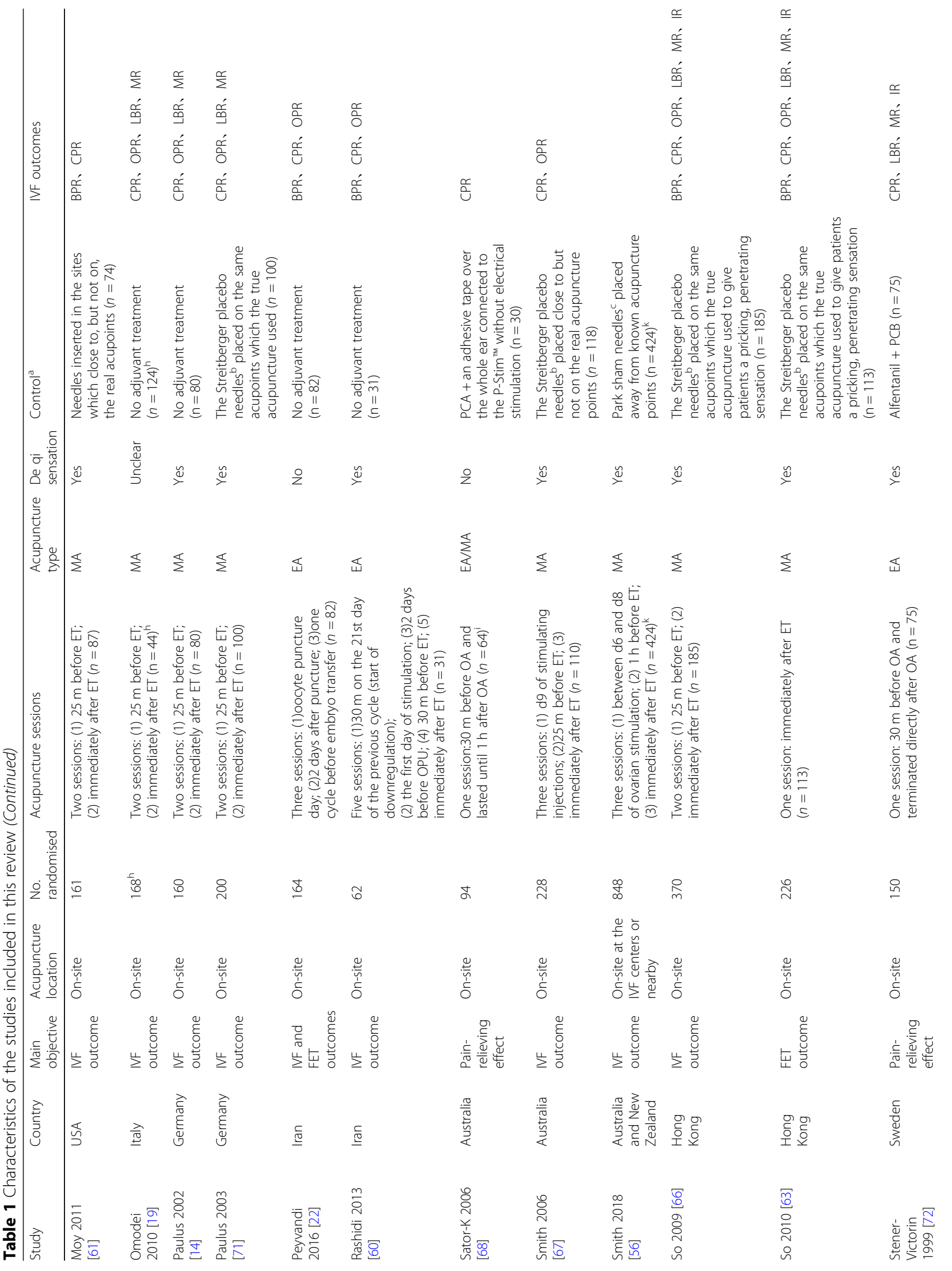




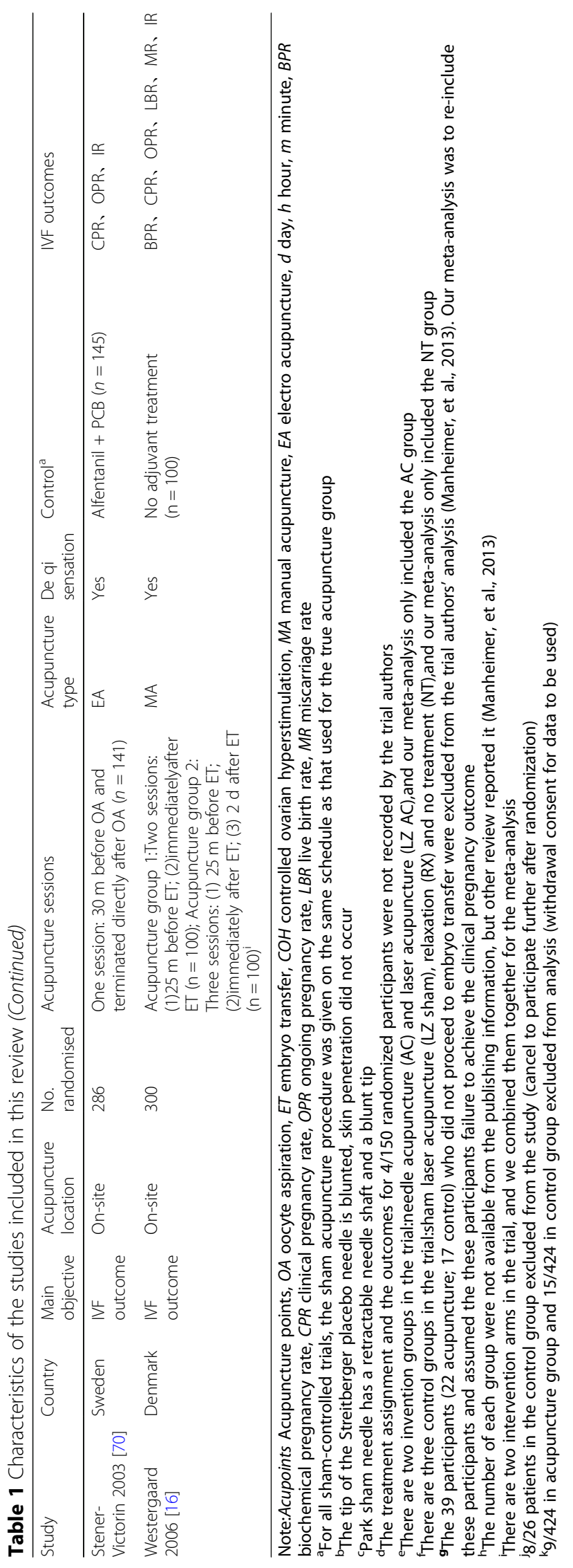




\section{Controls}

The control groups included no acupuncture and sham (placebo) acupuncture (Table 1). Eleven trials used sham or placebo acupuncture $[15,20,21,56,61,63,65-68$, $71]$, the others used no acupuncture intervention as the control group. One [21] had two control groups, one sham acupuncture group and one no acupuncture control group, and these were grouped separately for relevant subgroup analyses.

\section{IVF outcomes}

Clinical pregnancy rates were available from all the 27 trials. Fifteen [14-16, 18, 19, 56, 57, 59, 62-66, 71, 72] reported live birth rates and miscarriage rates.

\section{Methodological quality of the studies}

A summary of the risks of bias in included studies is presented in Fig. 1. Nineteen studies were at low risk of selection bias related to the random sequence generation. Eight studies $[17-19,22,62,69,70,72]$ were at unclear randomizations as they did not describe the randomization method used. For three studies $[18,57,64]$, there was inadequate allocation concealment, which placed them at high risk of bias. Six studies did not describe allocation concealment and were at unclear risk of this bias $[17,19,22,60,62,68]$. However, selection bias does not appear to be an issue in these trials, because of the similar baseline among groups. Owing to the nature of acupuncture studies, absolute double blinding was often not possible. Ten trials $[15,20,56,61,63$, $65-68,71]$ were at low risk of performance bias due to the use of sham or placebo acupuncture controls for blinding. Villahermosa et al. 2013 [21] had two control groups, a sham acupuncture group and a no acupuncture intervention control group, so we scored this criterion as "unclear risk" for blinding of patients. The remaining trials used no acupuncture intervention as the control group and were considered to be at high risk of bias. In nine trials $[16,17$, $56,59,60,64,67,69,70]$, some randomized women began the IVF process but did not complete embryo transfer, nevertheless, we included these women in the meta-analysis. In ten trials $[16,17,29,56,58,59,61,68$, $70,72]$, there were small numbers of randomized women with missing clinical pregnancy outcomes, however, we re-included them in the sensitivity analysis to explore its effect size on pregnancy outcomes.

\section{Effects of interventions}

CPR data (Fig. 2) were available from 27 trials $(n=6116)$. Pooled results showed a significant difference between

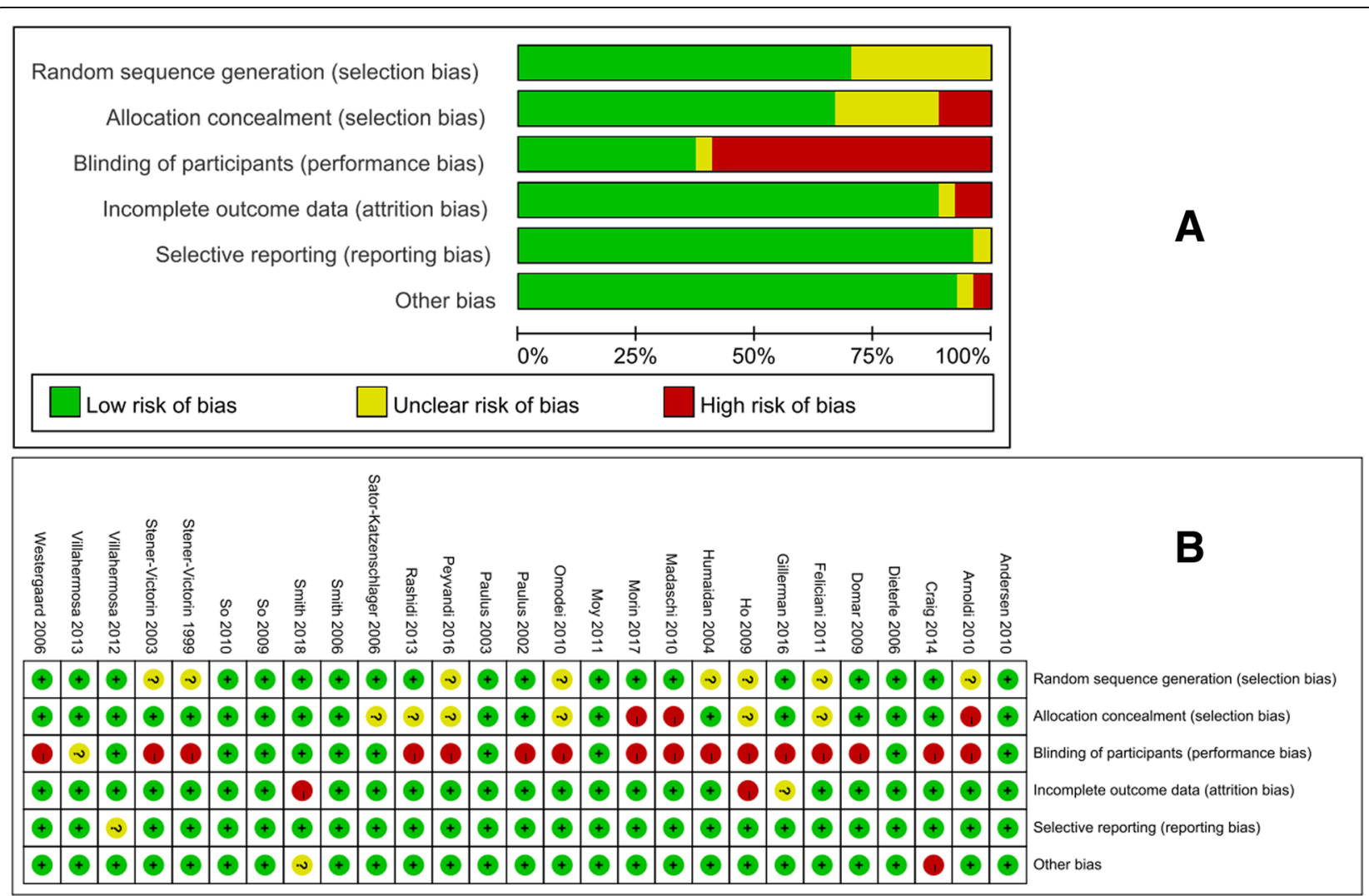

Fig. 1 Risk of bias graph (a) and risk of bias summary (b) based on review authors' judgments about each risk of bias item 


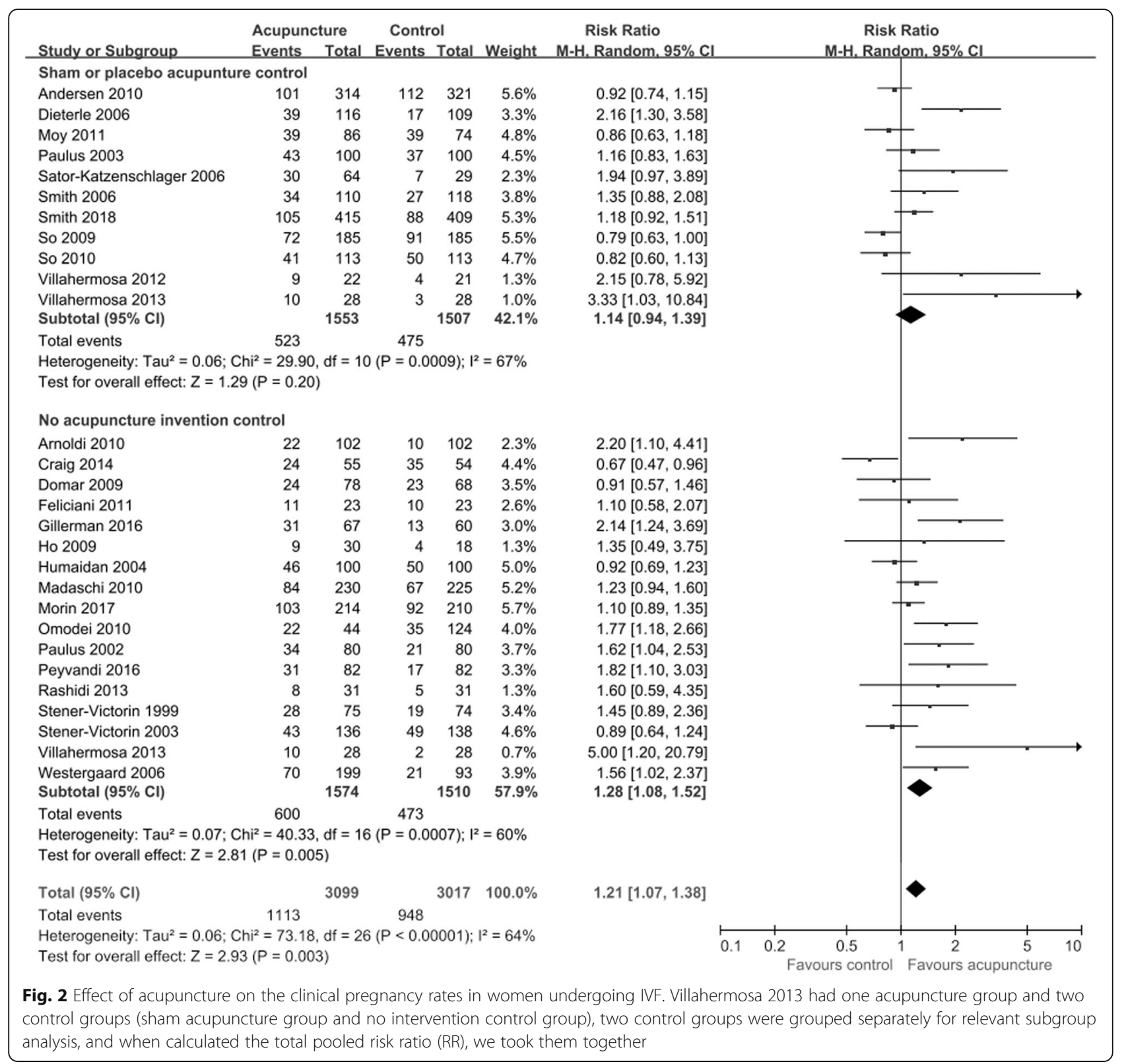

acupuncture and control groups $(\mathrm{RR}=1.21,95 \% \mathrm{CI}: 1.07-$ $\left.1.38, p=0.003, I^{2}=64 \%\right)$.

LBR data (Fig. 3) were available from15 trials $(n=$ 4472). There was no statistically significant difference between acupuncture and control groups $(R R=1.14$, 95\% CI: $\left.0.96-1.35, p=0.14, I^{2}=63 \%\right)$.

\section{Subgroup analysis}

There was statistical heterogeneity for pregnancy outcomes across studies (CPR: $\chi^{2} P$-value $<0.00001, I^{2}=64 \%$ and LBR: $\chi^{2} P$-value $=0.0005, I^{2}=63 \%$, respectively). Random-effects meta-regression and subgroup analyses were used to explore the potential sources of heterogeneity. The information on the variables for meta-regression analyses are displayed in Table 2. Meta-regression subgroup analyses were performed based on age, duration of infertility, primary infertility proportion, repeated IVF cycle proportion, number of embryos transferred, type of acupuncture invention, number of acupuncture treatments and type of control group to determine the possible effects of these variables.

Repeated IVF cycle proportion (number of women with a history of unsuccessful IVF attempt before included divided by number of women included in each trial) was reported in 11 studies $[15,18,21,56,63,65-$ $68,70,72]$. In subgroups classified by repeated IVF cycle proportion ( $\geq 50 \%$ or $<50 \%$ ), a significant outcome was found in the repeated IVF cycle proportion $\geq 50 \%$ group 


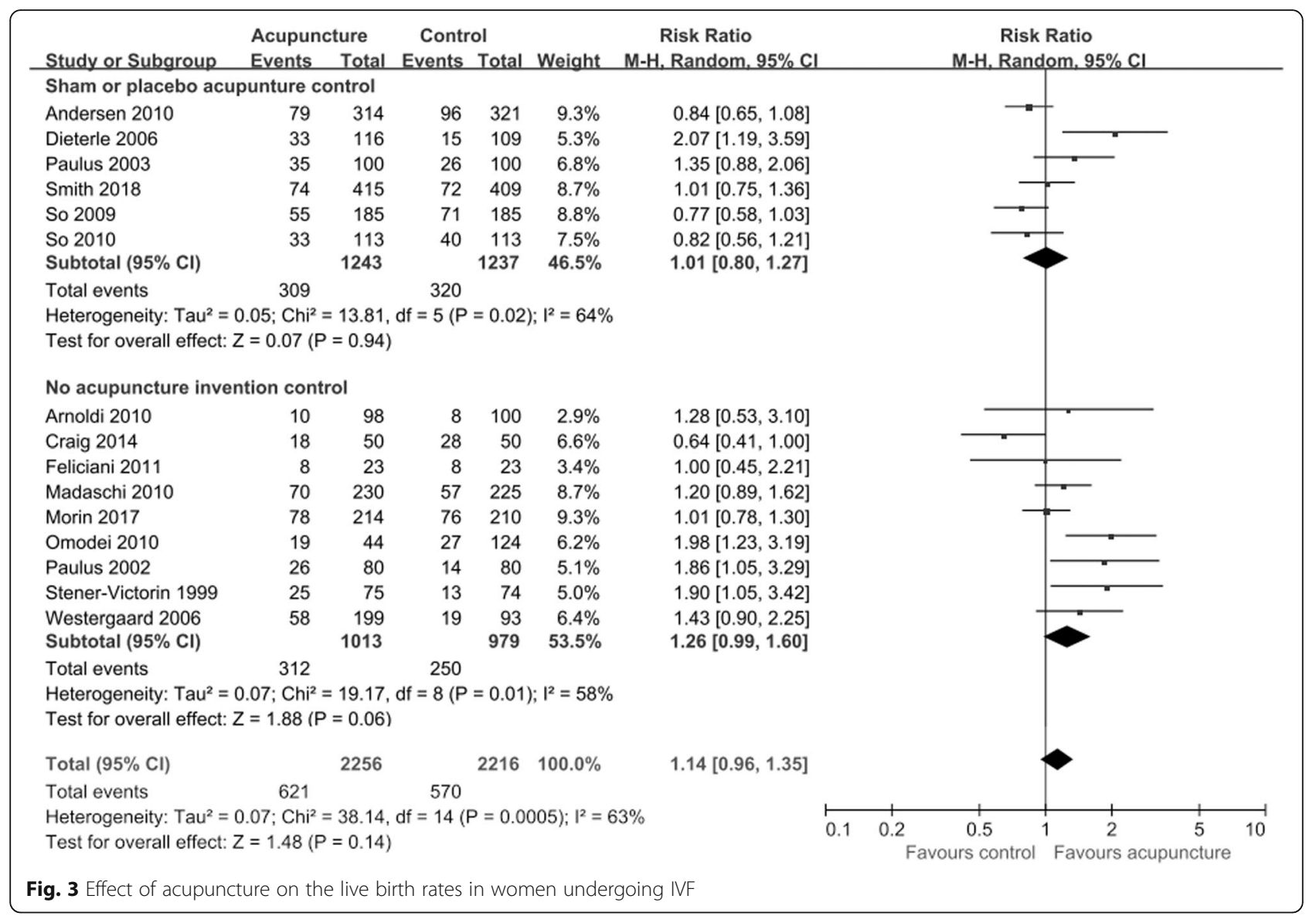

(CPR: $R R=1.60,95 \%$ CI: $1.28-2.00$; LBR: $R R=1.42,95 \%$ CI: 1.05-1.92, Table 3). The results of univariate meta-regressions indicated that pooled RR significantly varied with repeated IVF cycle proportion, and a positive correlation between the two was detected (Fig. 4). It was also the major sources of heterogeneity explained the great mass of the between-study variance for pregnancy outcomes (CPR: $p<0.001$, adjusted $R^{2}=100 \%, I^{2}$ residual $=0 \%$; LBR: $p=0.046$, adjusted $R^{2}=87.90 \%, I^{2}$ residual $=32.78 \%$; Table 3 ).

In the subgroup analysis based on the number of acupuncture treatments, the results indicated that women receiving two or more treatments had a significant improvement in clinical pregnancy (CPR: $R R=1.60,95 \%$ CI: 1.32-1.92, Table 3). The results of univariate meta-regression with clinical pregnancy rates as the dependent variables also suggested that number of acupuncture treatments was a statistically significant variable which explained $51.90 \%$ of the heterogeneity between included studies $(p=0.002$, adjusted $R^{2}=51.90 \%, I^{2}$ residual $=48.40 \%$, Table 3$)$. Unexpectedly, the meta-regression for live birth rates did not offer sufficient evidence to convince us that the number of acupuncture treatments was a covariate that led to heterogeneity ( $p=0.654$, adjusted $R^{2}=-7.71 \%, I^{2}$ residual $=64.01 \%$, Table 3 ), as per our hypothesis that there was an increasing gradient of RR with increasing acupuncture treatment number.

In addition, differences in age, duration of infertility, type of infertility, number of embryos transferred, type of acupuncture invention and control fail to explain a large proportion of the between trial variability. Detailed results of meta-regression subgroup analyses are displayed in Table 3.

\section{Side effects}

Fifteen trials evaluated the possible impact of acupuncture on miscarriage rates. Using the random-effects model, pooling of the results from the 15 trials showed no significant difference in the miscarriage outcome between the acupuncture and control groups $(R R=1.14$, 95\% CI: 0.93-1.41; Additional file 2: Figure S2).

\section{Sensitivity analyses}

The analyses described above were repeated under the four scenarios: (1) when we included randomized participants whose clinical pregnancy outcome data were missing, the above finding were not affected; (2) when 
Table $\mathbf{2}$ The information of variables for meta-regression subgroup analyses

\begin{tabular}{|c|c|c|c|c|c|c|c|c|}
\hline Study & $\begin{array}{l}\text { Age } \\
\text { (years) }\end{array}$ & $\begin{array}{l}\text { Duration of } \\
\text { infertility (years) }\end{array}$ & $\begin{array}{l}\text { Repeated IVF } \\
\text { cycles(\%) }\end{array}$ & $\begin{array}{l}\text { No. of embryos } \\
\text { transferred }\end{array}$ & $\begin{array}{l}\text { Primary } \\
\text { infertility(\%) }\end{array}$ & $\begin{array}{l}\text { No. of acupuncture } \\
\text { sessions }\end{array}$ & $\begin{array}{l}\text { Acupuncture } \\
\text { type }\end{array}$ & Control \\
\hline Andersen 2010 [65] & 31.0 & 2.5 & $42.4 \%$ & 1.39 & $71 \%$ & 1 & MA & $\mathrm{SC}$ \\
\hline Arnoldi 2010 [18] & & & $100 \%$ & 2.10 & & 2 & MA & NC \\
\hline Craig 2014 [59] & 33.1 & & & 2.10 & & 1 & MA & NC \\
\hline Dieterle 2006 [15] & 34.9 & 5.4 & $83.6 \%^{\mathrm{a}}$ & 2.60 & $80 \%$ & 2 & MA & SC \\
\hline Domar 2009 [29] & 36.1 & & & 2.51 & & 1 & MA & NC \\
\hline Feliciani 2011 [62] & 34.9 & & & 1.84 & & 3 & MA & NC \\
\hline Gillerman 2016 [58] & & & & & & 3 & MA & NC \\
\hline Ho 2009 [17] & 35.0 & & & 3.56 & & 4 & EA & NC \\
\hline Humaidan 2004 [69] & 31.0 & & & & & 1 & EA & NC \\
\hline Madaschi 2010 [64] & 35.0 & & & 2.15 & & 1 & MA & NC \\
\hline Morin 2017 [59] & 34.5 & & & 2.57 & & 1 & MA & NC \\
\hline Moy 2011 [61] & 33.2 & & & 2.15 & $63 \%$ & 1 & MA & SC \\
\hline Omodei 2010 [19] & 35.7 & & & 2.05 & & 1 & MA & NC \\
\hline Paulus 2002 [14] & 32.5 & & & 2.15 & & 1 & MA & NC \\
\hline Paulus 2003 [71] & 32.6 & & & & & 1 & MA & SC \\
\hline Peyvandi 2016 [22] & & & & & & 3 & EA & NC \\
\hline Rashidi 2013 [60] & 31.6 & 9.3 & & 2.55 & $76 \%$ & 4 & EA & NC \\
\hline Sator-K 2006 [68] & 33.8 & & $77.7 \%$ & & & 1 & EA/MA & SC \\
\hline Smith 2006 [67] & 36.0 & 2.9 & $52.2 \%$ & & $76 \%$ & 2 & MA & SC \\
\hline Smith 2018 [56] & 35.4 & & $70 \%$ & 1.32 & $74 \%$ & 2 & MA & SC \\
\hline So 2009 [66] & 36.0 & 4.0 & $30.5 \%$ & 1.89 & $61 \%$ & 1 & MA & SC \\
\hline So 2010 [63] & 36.0 & 5.0 & & 1.93 & $38 \%$ & 1 & MA & SC \\
\hline Stener-Victorin 1999 [72] & 33.4 & & $60.4 \%$ & 2.03 & & 1 & EA & NC \\
\hline Stener-Victorin 2003 [70] & 32.9 & & $47.8 \%$ & 1.92 & & 1 & EA & NC \\
\hline Villahermosa 2012 [20] & 34.2 & 4.0 & & & & 4 & MA & SC \\
\hline Villahermosa 2013 [21] & 36.2 & 4.5 & $100 \%$ & 2.13 & $82 \%$ & 4 & MA & $\mathrm{SC} / \mathrm{OC}^{\mathrm{b}}$ \\
\hline Westergaard 2006 [16] & 37.0 & 3.7 & $73.6 \%$ & 2.07 & $42 \%$ & $1 / 2^{c}$ & MA & NC \\
\hline
\end{tabular}

Note: MA manual acupuncture, EA electro acupuncture, SC sham control, NC no intervention control

${ }^{a}$ Dieterle 2006 reported clinical pregnancy rates for first, second and more than two IVF cycles, and then we grouped these three arms separately for meta-regression subgroup analyses

${ }^{\mathrm{b}}$ This trial had two control groups, sham acupuncture group and no intervention control group,and these groups were grouped separately for relevant subgroup analysis

'Westergarrd 2006 had two acupuncture treatment arms, one arm received two sessions and the other arm received three, and these arms were grouped separately for this meta-regression

acupuncture protocol which performed pre- and postET was regarded as two acupuncture sessions, the results for subgroup analysis and meta-regression remain unchanged (CPR: $p=0.008$; LBR: $p=0.817$ ); (3) when we restricted CPR to the 15 studies which reported LBR, the pooled results returned no difference between acupuncture and control groups for CPR (Additional file 3: Figure S3). In meta-regression and subgroup analyses, the repeated IVF cycle proportion remained a statistically significant effect modifier $(p=0.006)$, but the number of acupuncture treatments did not ( $p=0.240$; Additional file 5: Table S2); (4) we also analyzed sham-controlled and no adjuvant treatment controlled trials separately. However, since there were no large or significant differences between these two subsets in LBR (Table 3), as well as the largely similar subgroup effects across the two control groups, we preferred to conduct meta-regression and subgroup analyses stratified by control type for CPR. There were no significant changes in the results: the repeated IVF cycle proportion and number of acupuncture treatments remained statistically significant effect modifiers whenever restricted to no acupuncture invention control $(p=0.049$ and 0.035$)$ or sham (placebo) acupuncture control $(p=0.009$ and 0.010; Additional file 6: Table S3). 
Table 3 The results of meta-regression subgroup analyses for primary outcomes

\begin{tabular}{|c|c|c|c|c|c|c|c|c|c|}
\hline \multirow[t]{3}{*}{ Characteristic } & \multicolumn{5}{|c|}{ Subgroup analyses } & \multicolumn{4}{|c|}{ Meta-regression } \\
\hline & \multirow{2}{*}{$\begin{array}{l}\text { No. of } \\
\text { subjects }\end{array}$} & \multirow{2}{*}{$\begin{array}{l}\text { No. of } \\
\text { studies }\end{array}$} & \multirow{2}{*}{$\begin{array}{l}\text { Random-effects } \\
\text { RR (95\% CI) }\end{array}$} & \multicolumn{2}{|c|}{ Heterogeneity } & \multirow[t]{2}{*}{$\overline{\text { Coefficient }}$} & \multirow{2}{*}{$\begin{array}{l}p^{-} \\
\text {value }\end{array}$} & \multirow[t]{2}{*}{$1_{\text {resid }}^{2}$} & \multirow[t]{2}{*}{ Adj $R^{2}$} \\
\hline & & & & $\mathrm{P}^{2}$ & $P$ & & & & \\
\hline \multicolumn{10}{|l|}{ Clinical pregnancy } \\
\hline \multicolumn{10}{|l|}{ Age } \\
\hline$<33.3$ years & 1800 & 8 & $0.96(0.82,1.13)$ & $42.1 \%$ & 0.097 & 0.045 & 0.270 & $60.38 \%$ & $0.71 \%$ \\
\hline$\geq 33.3$ years & 3821 & 16 & $1.27(1.08,1.50)$ & $62 \%$ & $<0.005$ & & & & \\
\hline \multicolumn{10}{|l|}{ Duration of infertility } \\
\hline$<5.6$ years & 2103 & 8 & $1.27(0.95,1.71)$ & $77.6 \%$ & $<0.001$ & 0.072 & 0.523 & $76.70 \%$ & $-5.06 \%$ \\
\hline$\geq 5.6$ years & 62 & 1 & $1.60(0.59,4.35)$ & / & / & & & & \\
\hline \multicolumn{10}{|l|}{ Percentage of primary infertility } \\
\hline$<50 \%$ & 518 & 2 & $1.11(0.59,2.10)$ & $82.7 \%$ & 0.016 & 1.252 & 0.203 & $71.19 \%$ & $2.77 \%$ \\
\hline$\geq 50 \%$ & 2588 & 8 & $1.18(0.92,1.52)$ & $73.7 \%$ & $<0.001$ & & & & \\
\hline \multicolumn{10}{|l|}{ Percentage of repeated IVF cycle } \\
\hline$<50 \%$ & 1279 & 3 & $0.86(0.75,1.00)$ & $0 \%$ & 0.63 & 1.59 & $<0.001$ & $0 \%$ & $100 \%$ \\
\hline$\geq 50 \%$ & 2099 & 8 & $1.60(1.28,2.00)$ & $39 \%$ & 0.12 & & & & \\
\hline \multicolumn{10}{|l|}{ No. of embryos transferred } \\
\hline$<1.9$ & 1875 & 4 & $0.96(0.79,1.16)$ & $46 \%$ & 0.14 & 0.219 & 0.305 & $65.92 \%$ & $0.79 \%$ \\
\hline$\geq 1.9$ & 3186 & 16 & $1.24(1.03,1.49)$ & $67.3 \%$ & $<0.001$ & & & & \\
\hline \multicolumn{10}{|l|}{ Type of acupunture invention } \\
\hline Electroacupuncture & 958 & 7 & $1.30(0.96,1.75)$ & $56.5 \%$ & 0.032 & 0.066 & 0.713 & $65.29 \%$ & $-6.38 \%$ \\
\hline Manual acupuncture & 5187 & 21 & $1.21(1.04,1.40)$ & $67.8 \%$ & $<0.001$ & & & & \\
\hline \multicolumn{10}{|l|}{ No. of acupuncture treatments } \\
\hline one session & 3962 & 16 & $1.06(0.92,1.21)$ & $61.1 \%$ & 0.001 & 0.248 & 0.002 & $48.40 \%$ & $51.90 \%$ \\
\hline$\geq$ two sessions & 2055 & 12 & $1.60(1.32,1.92)$ & $24.9 \%$ & 0.199 & & & & \\
\hline \multicolumn{10}{|l|}{ Type of control group } \\
\hline Sham or placebo acupuncture control & 3060 & 11 & $1.14(0.94,1.39)$ & $66.6 \%$ & 0.001 & -0.109 & 0.466 & $62.34 \%$ & $-0.25 \%$ \\
\hline No acupuncture invention control & 3084 & 17 & $1.28(1.081 .52)$ & $60.3 \%$ & 0.001 & & & & \\
\hline \multicolumn{10}{|l|}{ Live birth } \\
\hline \multicolumn{10}{|l|}{ Age } \\
\hline$<33.3$ years & 895 & 3 & $0.96(0.59,1.58)$ & $77 \%$ & 0.013 & 0.026 & 0.693 & $68.74 \%$ & $-13.18 \%$ \\
\hline$\geq 33.3$ years & 3179 & 10 & $1.18(0.96,1.45)$ & $63 \%$ & 0.003 & & & & \\
\hline Duration of infertility & & & & & & & & & \\
\hline$<5.6$ years & 1748 & 5 & $1.03(0.76,1.40)$ & $71.6 \%$ & 0.007 & 0.157 & 0.712 & $75.35 \%$ & $-20.26 \%$ \\
\hline$\geq 5.6$ years & / & / & / & / & / & & & & \\
\hline Percentage of primary infertility & & & & & & & & & \\
\hline$<50 \%$ & 518 & 2 & $1.07(0.62,1.83)$ & $69.7 \%$ & 0.069 & 0.497 & 0.658 & $71.40 \%$ & $-43.04 \%$ \\
\hline$\geq 50 \%$ & 2054 & 4 & $1.00(0.74,1.36)$ & $71.7 \%$ & 0.014 & & & & \\
\hline Percentage of repeated IVF cycle & & & & & & & & & \\
\hline$<50 \%$ & 1005 & 2 & $0.81(0.67,1.01)$ & $0 \%$ & 0.673 & 1.256 & 0.046 & $32.78 \%$ & $87.90 \%$ \\
\hline$\geq 50 \%$ & 1688 & 5 & $1.42(1.05,1.92)$ & $45 \%$ & 0.12 & & & & \\
\hline No. of embryos transferred & & & & & & & & & \\
\hline$<1.9$ & 1865 & 4 & $0.87(0.74,1.02)$ & $0 \%$ & 0.604 & 0.386 & 0.163 & $62.55 \%$ & $7.83 \%$ \\
\hline$\geq 1.9$ & 2397 & 10 & 1. $27(1.00,1.62)$ & $66.0 \%$ & 0.002 & & & & \\
\hline
\end{tabular}


Table 3 The results of meta-regression subgroup analyses for primary outcomes (Continued)

\begin{tabular}{|c|c|c|c|c|c|c|c|c|c|}
\hline \multirow[t]{3}{*}{ Characteristic } & \multicolumn{5}{|c|}{ Subgroup analyses } & \multicolumn{4}{|c|}{ Meta-regression } \\
\hline & \multirow{2}{*}{$\begin{array}{l}\text { No. of } \\
\text { subjects }\end{array}$} & \multirow{2}{*}{$\begin{array}{l}\text { No. of } \\
\text { studies }\end{array}$} & \multirow{2}{*}{$\begin{array}{l}\text { Random-effects } \\
\text { RR }(95 \% \mathrm{Cl})\end{array}$} & \multicolumn{2}{|c|}{ Heterogeneity } & \multirow[t]{2}{*}{ Coefficient } & \multirow{2}{*}{$\begin{array}{l}p^{-} \\
\text {value }\end{array}$} & \multirow[t]{2}{*}{$I_{\text {resid }}^{2}$} & \multirow[t]{2}{*}{ Adj $R^{2}$} \\
\hline & & & & $1^{2}$ & $P$ & & & & \\
\hline \multicolumn{10}{|l|}{ Type of acupunture invention } \\
\hline Electroacupuncture & 149 & 1 & $1.90(1.05,3.42)$ & / & / & 0.534 & 0.226 & $61.92 \%$ & $9.04 \%$ \\
\hline Manual acupuncture & 4323 & 14 & $1.11(0.93,1.31)$ & $62.0 \%$ & 0.001 & & & & \\
\hline \multicolumn{10}{|l|}{ No. of acupuncture treatments } \\
\hline one session & 3080 & 11 & $1.13(0.92,1.39)$ & $70.4 \%$ & $<0.001$ & 0.099 & 0.654 & $64.01 \%$ & $-7.71 \%$ \\
\hline$\geq$ two sessions & 1485 & 5 & $1.22(0.93,1.60)$ & $23.2 \%$ & 0.266 & & & & \\
\hline \multicolumn{10}{|l|}{ Type of control group } \\
\hline Sham or placebo acupuncture control & 2480 & 6 & $1.01(0.80,1.27)$ & $63.8 \%$ & 0.017 & -0.213 & 0.274 & $60.40 \%$ & $7.21 \%$ \\
\hline No acupuncture invention control & 1992 & 9 & $1.26(0.99,1.60)$ & $58.3 \%$ & 0.014 & & & & \\
\hline
\end{tabular}
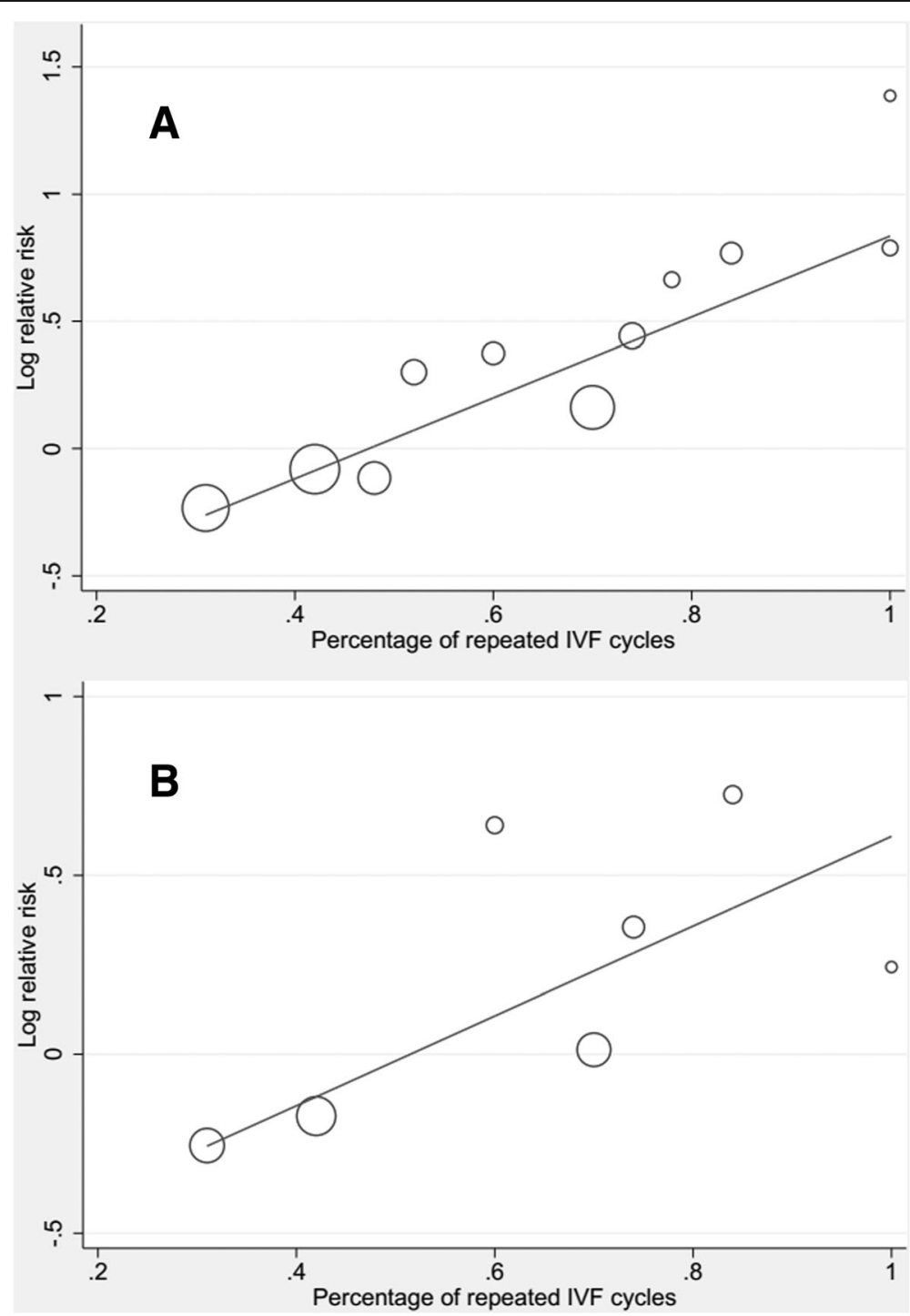

Fig. 4 Meta-regression between the effects of acupuncture on clinical pregnancy rates (a)/ live birth rates (b) and repeated IVF cycle proportion as a single continuous covariate 
In addition, we performed the meta-regression subgroup analyses according to 'risk of bias' of included studies. When we restricted study eligibility to "low risk of bias", the results remained stable (Additional file 7: Table S4).

\section{Publication bias}

Funnel plot analysis showed that there were no significant publication biases for most analyses, except for acupuncture compared with all controls for clinical pregnancy rates (Fig. 5). Both Begg's and Egger's test results supported the possible of publication bias $(p=0.006)$.

\section{Levels of evidence}

Overall, the levels of evidence as determined by GRADE were very low for the pooled CPR (27 studies) and LBR (15 studies) from all the acupuncture groups compare with control groups. For miscarriage rates, moderate quality of evidence was found. In stratified analyses, moderate quality of evidence showed that acupuncture improved IVF outcomes among women with a history of unsuccessful IVF attempt. However, the levels of evidence for other subgroup analyses were found to be from very low to low.

\section{Discussion}

\section{Main findings}

The objective of this review was to summarize and evaluate the effects of acupuncture on pregnancy outcomes among women undergoing IVF. Compared with controls, we found a low level of evidence that acupuncture significantly increased CPR among women undergoing IVF $(\mathrm{RR}=1.21,95 \% \mathrm{CI}$ : $1.07-1.38)$, but not LBR. However, there was substantial heterogeneity between these trials. Subsequently, in subgroup analyses classified by the repeated IVF cycle proportion $(\geq 50 \%$ or $<50 \%$ ) and number of acupuncture treatments, we found a moderate level of evidence that acupuncture improved IVF outcomes in repeated IVF cycle proportion $\geq 50 \%$ subset (CPR: $R R=1.60,95 \% \mathrm{CI}: 1.28-2.00$; LBR: $\mathrm{RR}=1.42$, 95\% CI: $1.05-1.92$ ), as well as in more than one acupuncture treatment subset for $C P R(R R=1.60$, 95\% CI: 1.32-1.92). And the univariate meta-regression suggested a positive correlation between the pooled RR and repeated IVF cycle proportion (CPR: $p<0.001$; LBR: $p=0.046$ ), it was also the major sources of heterogeneity (CPR: adjusted $R^{2}=100 \%$; LBR: adjusted $R^{2}=87.90 \%$ ). Similar results were found between the pooled CPR and number of acupuncture treatments in the meta-regression model $\left(p=0.002\right.$, adjusted $R^{2}=51.90 \%, I^{2}$ residual $=48.40 \%)$.

Moreover, the results of sensitivity analysis were also consistent with the findings described above, except for the scenario when CPR results were restricted to the 15 studies which reported LBR. In that case the results of the meta-regression between the pooled CPR and number of acupuncture treatments turned negative.

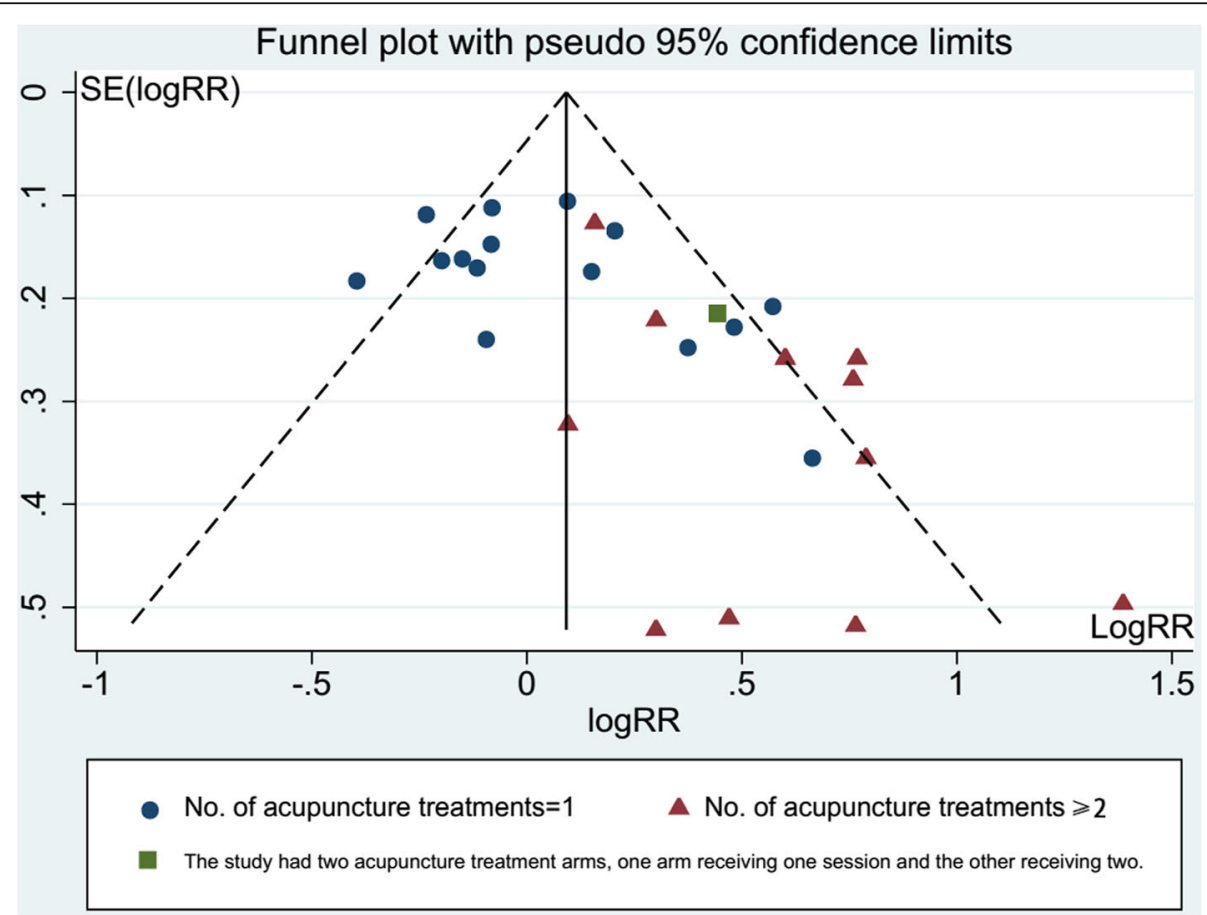

Fig. 5 The funnel plot for the 27 eligible studies (Begg's and Egger's test, $p=0.006$ ). The studies are separated into two groups by No. of acupuncture treatments either one or more than one 


\section{Interpretation}

This review indicates that acupuncture improves CPR rather than LBR. Firstly, we considered this suggested a higher risk of miscarriage. Fifteen trials evaluated the possible impact of acupuncture on miscarriage rates, however, we did not find statistical evidence for this (RR =1.14, 95\% CI: 0.93-1.41; Additional file 2: Figure S2). Secondly, it may be caused by the missing information of LBR. Only 15 among 27 studies reported both CPR and LBR, when we restricted CPR to the 15 studies which reported LBR, the pooled results returned no difference between acupuncture and control groups for CPR (Additional file 3: Figure S3). One plausible reason for this variation could be the heterogeneity (population-, treatment- and study design-related varieties) among trials. In our subgroup analyses and sensitivity analyses, we found a significantly increased CPR and LBR in two or more acupuncture treatments and the repeated IVF cycle proportion $\geq 50 \%$ subsets, but not in one acupuncture treatment and $<50 \%$ subsets (Table 3 ), and when we restricted CPR results to the 15 studies which reported LBR, the results remained stable (Additional file 5: Table S2). Therefore, heterogeneity among trials may be one of possible explanations for the difference of statistical significance between CPR and LBR.

\section{Meta-regression subgroup analyses based on clinical characteristics}

We found that patients with repeated IVF cycles benefit more from acupuncture than do women with first IVF cycle acupuncture. Repeated IVF cycle usually implies at least one implantation failure, which may be a consequence of embryo or endometrial factors [74-77], as well as the increased vulnerability to stress [78-80]. From a Chinese medical perspective, the primary principles of fertility treatment with acupuncture are to harmonize the function of the internal organs, clear obstructions and improve the qi and blood supply within the abdominal environment in order to improve egg quality and the receptiveness of the endometrium [81]. On one hand, numerous studies have shown that acupuncture can stimulate ovulation by adjusting endocrine function of the hypothalamic-pituitary-ovarian axis in women with an ovulation disorder [24, 82-84], as well as improve egg quality $[60,85]$, which may related to a beneficial regulation of TNF- $\alpha$ levels [85]. On the other hand, acupuncture improves blood circulation to the uterus and ovaries by inhibiting uterine central sympathetic nerve activity, and thereby optimizing endometrial receptivity [17, 27, 86, 87]. Moreover, acupuncture may be a useful intervention to reduce infertility-related stress and depression by changing the stress hormones (serum cortisol and prolactin) [28-30, 88-91]. Thus, as a corollary, women undergoing repeated IVF cycles may benefit more from acupuncture, which is consistent with our meta-analysis. However, the exact mechanism of this effect is still poorly understood. As repeated IVF cycle is a proxy for implantation failure, which associated with age, duration and cause of infertility, number of embryos transferred, embryo quality [48, 92-96], future studies should further investigate the relationship between these parameters and the efficacy of acupuncture on IVF outcomes.

Although meta-regression subgroup analyses provided insufficient evidence to support hypotheses regarding the age, duration of infertility and number of embryos transferred, this may due to aggregation bias. These data were extracted in the form of mean values, which can only be summarized at the level of the individual study. This makes the effect of a characteristic not always identifiable due to the ecological fallacy. For example, there may be a strong relationship between age and treatment effect that is apparent within each study, however, if the mean ages for the trials are similar, then no relationship will be apparent by looking at trial mean ages and trial-level effect estimates [47]. Analyses of pooled, patient-level data will be useful to find the true role of these parameters on the efficacy of acupuncture in future studies.

\section{Meta-regression subgroup analyses based on methodological characteristics}

The quality of included studies is an influential factor in our meta-analysis. In our review, we found a statistically significant subgroup effect for the type of control on CPR, suggesting some placebo effect. As our outcomes are entirely objective, and are unlikely to be affected by placebo effects [97-100], we also consider explanations such as heterogeneity among trials. Therefore, we conducted subgroup analyses stratified by the number of acupuncture treatments in addition to control type for CPR. However, the pooled results showed no difference between sham (placebo) acupuncture control and no acupuncture invention control when restricted to studies which performed one acupuncture treatment (95\% CI: $0.79-1.09$ and $0.95-1.36)$, or more than one acupuncture treatment (95\% CI: $1.13-2.17$ and 1.36-2.15). This suggests a minor contribution of placebo effect for IVF outcomes.

Recent studies have shown that sham and placebo acupuncture are sometimes therapeutically effective [101103]. Sham or placebo acupuncture usually consists of non-insertion or superficial insertion in related acupoints or needle insertion at unrelated acupoints or non-acupoints. However, all evoke activity in cutaneous afferent nerves $[101,102]$. It has been reported that placebos have small benefits in studies where continuous subjective outcomes are measured (especially patient- 
reported outcomes), but there are no significant effects on objective or binary outcomes [97-100]. More accurately, for objectively measured outcome parameters, placebo effects can improve physical processes (e.g., blood flow to the uterus) more easily and effectively than biochemical processes (e.g., endogenous hormone release) [104]. Consequently, it is more plausible to cautiously use sham acupuncture for subjective, patient-reported outcomes and objectively measured physical parameters before its use or non-use is established.

\section{Limitations}

Our study has a number of limitations. Firstly, the funnel plot of the effects of acupuncture on clinical pregnancy rates (Fig. 5) indicates some small-study effects - a tendency for the intervention effects estimated in smaller studies to differ from those estimated in larger studies [105]. The Begg's and Egger's test results $(p=0.006)$ support the finding of small study-effects that overestimate the treatment effect. However, the purpose of the test was not to test for publication bias alone, the reasons other than publication bias should also be considered, including other language bias, citation bias, time lag bias and multiple publication bias $[106,107]$. We imposed no restrictions on publication type or language of publication, scanned the digital databases, the conference proceedings and reference lists of relevant primary and review articles. In addition, we corresponded with study investigators to clarify further data, so it seems that the review is less likely to be subject to the other reported biases. According to the Cochrane guidelines, "true heterogeneity in treatment effect may also lead to funnel plot asymmetry" [107]. For example, substantial benefit may be seen only in patients at high risk for the outcome which is affected by the intervention [108, 109]. Furthermore, some interventions may have been implemented less thoroughly in larger trials, therefore, have resulted in smaller estimates of the intervention effect [110]. In our analysis, we found that women with a history of unsuccessful IVF attempt, as well as having received more than one acupuncture treatment, will benefit more from the effects of acupuncture on clinical pregnancy rates. Given that only 11 studies provided the date of repeated IVF cycle proportion, we therefore added the number of acupuncture treatments to the funnel plot, and the resulting plot shows that the trials with more than one acupuncture treatment indicate more positive results (Fig. 5). We also checked separate funnel plots and conducted corresponding separate Egger tests for one and more than one acupuncture treatment trial subgroups. Ultimately, no evidence of publication bias was found when restricting to one or more than one acupuncture treatment trial subset (Begg's and Egger's tests for one acupuncture treatment: $p=0.137$ and 0.07; for more than one acupuncture treatment: $p=0.451$ and 0.06 , respectively). Therefore, less thoroughly implemented or high risk patients for pregnancy outcomes affected by acupuncture may be one of many possible explanations for the funnel plot asymmetry. That is, asymmetry may be partly due to the existence of true heterogeneity. A cumulative meta-analysis was conducted to clarify the contribution of small-study effects on the results based on the sample size (Fig. 6). The cumulative effect size increased gradually after the first study with the biggest sample size (824), but reached a significant steady state at sample size 164 . However, analysis by excluding the studies with small sample sizes $(<100)$ still showed a statistically significant difference ( $R R=1.16,95 \% \mathrm{CI}: 1.02-1.32$ ). These results indicate a minor contribution of publication bias on effect sizes.

Secondly, there were 27 studies reporting clinical pregnancy outcomes, but only 15 among 27 studies reported live birth rates. Therefore, the deficiency of IVF outcomes may lead to substantial variation, which resulted in imprecise $R R$ and unclear associations between the strength of RR and related variables.

Lastly, the differences in acupuncture protocols were unidentified confounders which complicated the interpretation of the efficacy of acupuncture on IVF outcomes. For acupuncture time, several systematic reviews reported that IVF outcomes improved significantly when acupuncture was conducted during $\mathrm{COH}$, but not ET and OA [38, 41, 42]. Moreover, from a Chinese medicine perspective, acupoints should be chosen based on the different signs and symptoms of individuals. This may account for the results of Cui et al.'s study, where women with three different syndromes were treated with the same acupuncture protocol. They showed that the effects of acupuncture on IVF-ET in women with kidney deficiency type and liver-qi stagnation type were better than those of phlegm-dampness type [111]. In addition, the frequency, duration and the mode of stimulation of acupuncture treatment may also influence to the efficacy of acupuncture. Fortunately, a Delphi consensus protocol was published to establish the parameters of best acupuncture practice for ART in 2012. In future studies this protocol may be helpful to clarify the efficacy of acupuncture on IVF outcomes.

\section{Comparison with other studies and reviews}

We acknowledge that there are several systematic reviews and meta-analyses analyzed the effects of acupuncture among women undergoing IVF [31-43]. However, they showed differences in their results and conclusions. The reasons for these discrepancies may arise from the 


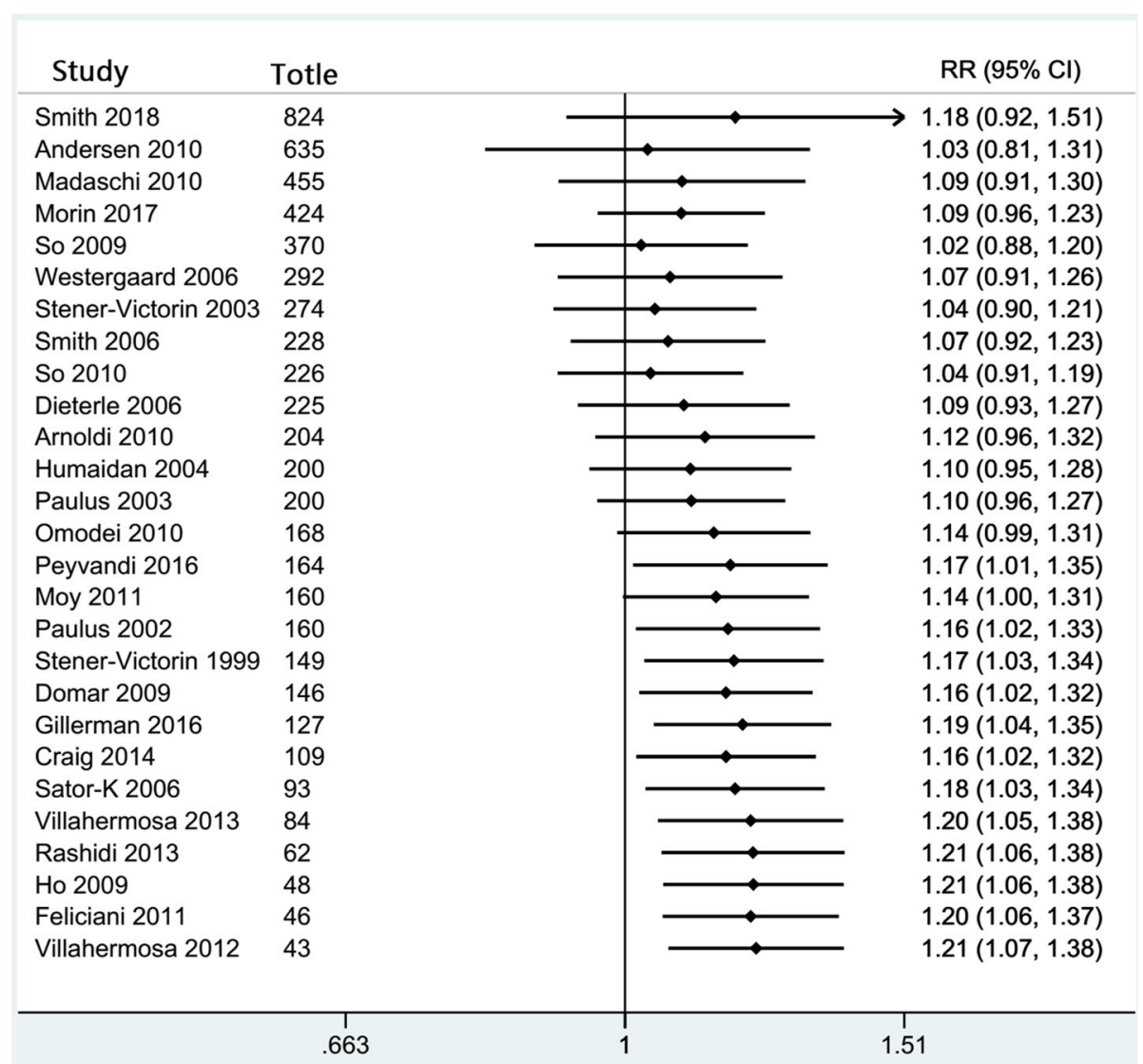

Fig. 6 The result of cumulative meta-analysis based on sample size

heterogeneity across studies, such as variation in participants, interventions, trial design and quality. And few studies try to investigate sources of heterogeneity. In our meta-analysis, we attempted pre-specified meta-regression subgroup analyses to explore the influences of eight variables on the effects of acupuncture on both clinical pregnancy and live birth outcomes. Ultimately, we revealed some new findings. First of all, there is a significant benefit of adjuvant acupuncture on both CPR and LBR for women with a history of unsuccessful IVF attempt. Secondly, number of acupuncture treatments is a promising influential factor for the effect estimates. In addition, our search identified four additional studies [22, 56-58], which were not included in the earlier reviews.

\section{Conclusion}

Our analysis finds a benefit of acupuncture for IVF outcomes among women with a history of unsuccessful IVF attempt, and number of acupuncture treatments is a potential influential factor. As repeated IVF cycle is possibly a proxy for implantation failure, which associated with poor prognoses (i.e. increasing age, longer history of infertility, elevated Peak FSH). In the future, analyses of pooled, patient-level data will be useful to find the true role of these parameters on the efficacy of acupuncture on IVF outcomes.

\section{Additional files}

Additional file 1: Figure S1. Study selection PRISMA flow diagram. (TIF $5172 \mathrm{~kb}$ )

Additional file 2: Figure S2. Effects of acupuncture on spontaneous abortion outcome. (TIF $1233 \mathrm{~kb}$ )

Additional file 3: Figure S3. Effects of acupuncture on clinical pregnancy rates for 15 studies which reported live birth rates. (TIF $1519 \mathrm{~kb}$ )

Additional file 4: Table S1. Search strategy for the MEDLINE Database. (DOC $50 \mathrm{~kb}$ )

Additional file 5: Table S2. The results of meta-regression subgroup analyses for the 15 studies which reported LBR. (DOC $61 \mathrm{~kb}$ )

Additional file 6: Table S3. The results of meta-regression subgroup analyses stratified by control type. (DOC $84 \mathrm{~kb}$ )

Additional file 7: Table S4. The results of meta-regression subgroup analyses according to 'risk of bias' for primary outcomes. (DOC $73 \mathrm{~kb}$ )

Additional file 8: Appendix 1. The reasons for exclusion studies. (DOC $175 \mathrm{~kb}$ ) 


\section{Abbreviations}

$\mathrm{Cl}$ : Confidence intervals; $\mathrm{COH}$ : Controlled ovarian hyperstimulation; CPR: Clinical pregnancy rate; DR: Delivery rates; EA: Electrical acupuncture; ET: Embryo transfer; ICSI: Intracytoplasmic sperm injection; IVF: In vitro fertilization; LBR: Live birth rate; MA: Manual acupuncture; PCOS: Polycystic ovary syndrome; RCTs: Randomized controlled trials; RR: Relative risks; TCM: Traditional Chinese medicine

\section{Acknowledgments}

We thank Bin Xu (Nanjing University of Chinese Medicine) and Qin Sun (Nanjing General Hospital of Nanjing Military Region) for providing useful suggestions during the preparation of the meta-analysis. And the authors would like to express.

their deep thanks to the following co-authors of included RCTs, for confirming and providing data related to their.

respective trials: LaTasha Craig MD, Alice Domar PhD and Jing Li MD.

\section{Funding}

Supported by grants from China National Natural Science Foundation (no. 81473767 to You-bing Xia) .

\section{Availability of data and materials}

The datasets supporting the conclusions of this article are included within the article.

\section{Authors' contributions}

ZYX, BY, ZHP and YBX conceived the review and determined inclusion eligibility of trials. ZYX, XL and PYY performed literature searches, study selection and data extraction. ZHP and ZYX assessed the quality of studies, contributed to the analysis and interpretation of the data. ZYX wrote the initial draft. ZYX, BY, ZHP, LC, YYM, JC, QL, and YBX critically revised the manuscript. All authors read and approved the final manuscript.

\section{Ethics approval and consent to participate}

Not applicable.

\section{Consent for publication}

Not applicable.

\section{Competing interests}

All authors declare that they have no competing interests.

\section{Publisher's Note}

Springer Nature remains neutral with regard to jurisdictional claims in published maps and institutional affiliations.

\section{Author details}

${ }^{1}$ Nanjing Hospital of Traditional Chinese Medicine, The Third Affiliated Hospital of Nanjing University of Chinese Medicine, Nanjing, China. ${ }^{2}$ Department of Epidemiology and Biostatistics, School of Public Health Nanjing Medical University, Nanjing, China. ${ }^{3}$ Center of Reproductive Medicine, Nanjing Jinling Hospital, Nanjing University School of Medical, Nanjing, China. ${ }^{4}$ The Second School Medical College, Nanjing University of Chinese Medicine, Nanjing, China. ${ }^{5}$ Changzheng Hospital of the Second Military Medical University, Nanjing branch, Nanjing, China. ${ }^{6}$ Nanjing Jiangning Hospital of Traditional Chinese Medicine, Nanjing, China. ${ }^{7}$ Xuzhou Medical University, Xuzhou, China.

Received: 28 June 2017 Accepted: 15 May 2019

Published online: 14 June 2019

\section{References}

1. Datta J, Palmer MJ, Tanton C, Gibson LJ, Jones KG, Macdowall W, Glasier A Sonnenberg $\mathrm{P}$, Field $\mathrm{N}$, Mercer $\mathrm{CH}$, et al. Prevalence of infertility and help seeking among 15000 women and men. Human reproduction (Oxford, England). 2016;31(9):2108-18.

2. Zegers-Hochschild F, Adamson GD, Dyer S, Racowsky C, de Mouzon J, Sokol R, Rienzi L, Sunde A, Schmidt L, Cooke ID, et al. The international glossary on infertility and fertility care, 2017. Fertil Steril. 2017;108(3):393-406.

3. Adamson GD, de Mouzon J, Chambers GM, Zegers-Hochschild F, Mansour R, Ishihara O, Banker M, Dyer S. International Committee for Monitoring
Assisted Reproductive Technology: world report on assisted reproductive technology, 2011. Fertil Steril. 2018;110(6):1067-80.

4. Kissin DM, Kawwass JF, Monsour M, Boulet SL, Session DR, Jamieson DJ. Assisted hatching: trends and pregnancy outcomes, United States, 20002010. Fertil Steril. 2014;102(3):795-801.

5. De Geyter C, Calhaz-Jorge C, Kupka MS, Wyns C, Mocanu E, Motrenko T, Scaravelli G, Smeenk J, Vidakovic S, Goossens V. ART in Europe, 2014: results generated from European registries by ESHRE: the European IVF-monitoring consortium (EIM) for the European Society of Human Reproduction and Embryology (ESHRE). Hum Reprod. 2018;33(9):1586-601.

6. ESHRE Capri Workshop Group. Economic aspects of infertility care: a challenge for researchers and clinicians. Hum Reprod. 2015;30(10):2243-8.

7. Chambers GM, Sullivan EA, Ishihara O, Chapman MG, Adamson GD. The economic impact of assisted reproductive technology: a review of selected developed countries. Fertil Steril. 2009:91(6):2281-94.

8. The cost of IVF: 4 things I learned while battling infertility [https://www. forbes.com/sites/learnvest/2014/02/06/the-cost-of-ivf-4-things-i-learnedwhile-battling-infertility/].

9. Fertility: assessment and treatment for people with fertility problems (update) costing report [www.nice.org.uk/guidance/CG156].

10. Vincent $C$. The safety of acupuncture: Acupuncture is safe in the hands of competent practitioners. BMJ. 2001;323(7311):467-8.

11. Witt CM, Pach D, Brinkhaus B, Wruck K, Tag B, Mank S, Willich SN. Safety of acupuncture: results of a prospective observational study with 229,230 patients and introduction of a medical information and consent form. Forsch Komplementmed. 2009;16(2):91-7.

12. Park J, Sohn Y, White AR, Lee $H$. The safety of acupuncture during pregnancy: a systematic review. Acupunct Med. 2014;32(3):257-66.

13. da Silva JB. Acupuncture in pregnancy. Acupunct Med. 2015;33(5):350-2

14. Paulus WE, Zhang M, Strehler E, El-Danasouri I, Sterzik K. Influence of acupuncture on the pregnancy rate in patients who undergo assisted reproduction therapy. Fertil Steril. 2002;77(4):721-4.

15. Dieterle S, Ying G, Hatzmann W, Neuer A. Effect of acupuncture on the outcome of in vitro fertilization and intracytoplasmic sperm injection: a randomized, prospective, controlled clinical study. Fertil Steril. 2006;85(5): 1347-51.

16. Westergaard LG, Mao Q, Krogslund M, Sandrini S, Lenz S, Grinsted J. Acupuncture on the day of embryo transfer significantly improves the reproductive outcome in infertile women: a prospective, randomized trial. Fertil Steril. 2006:85(5):1341-6.

17. Ho M, Huang LC, Chang YY, Chen HY, Chang WC, Yang TC, Tsai HD. Electroacupuncture reduces uterine artery blood flow impedance in infertile women. Taiwan J Obstet Gynecol. 2009;48(2):148-51.

18. Arnoldi M, Diaferia D, Corbucci MG, De Lauretis $L$. The role of acupuncture in patients at unfavourable reproductive prognosis in IVF: a prospective randomised study. Hum Reprod. 2010;25:i257.

19. Omodei U, Piccioni G, Tombesi S, Dordoni D, Fallo L, Ghilardi F. Effect of acupuncture on rates of pregnancy among women undergoing in vitro fertilization. Fertil Steril. 2010;94(4):S170.

20. Isoyama D, Cordts EB, van Niewegen A, de Carvalho WDP, Matsumura ST, Barbosa CP. Effect of acupuncture on symptoms of anxiety in women undergoing in vitro fertilisation: a prospective randomised controlled study. Acupunct Med. 2012;30(2):85-8.

21. Isoyama D, Santos LG, Nogueira MB, Vilarino FL, Barbosa CP. Influence of acupuncture on the outcomes of in vitro fertilisation when embryo implantation has failed: a prospective randomised controlled clinical trial. Acupunct Med. 2013;31(2):157-61.

22. Peyvandi S, Rokhgireh S, Esmaeeli N. A survey of acupuncture effect on the result of invitro fertilization. World J Acupuncture-Moxibustion. 2016;26(03):55-8.

23. Ferin $M$, Vande Wiele R. Endogenous opioid peptides and the control of the menstrual cycle. Eur J Obstet Gynecol Reprod Biol. 1984;18(5-6):365-73.

24. Chen BY. Acupuncture normalizes dysfunction of hypothalamic-pituitaryovarian axis. Acupunct Electrother Res. 1997;22(2):97-108.

25. Chang $\mathrm{R}$, Chung $\mathrm{PH}$, Rosenwaks $\mathrm{Z}$. Role of acupuncture in the treatment of female infertility. Fertil Steril. 2002;78(6):1149-53.

26. Anderson BJ, Haimovici F, Ginsburg ES, Schust DJ, Wayne PM. In vitro fertilization and acupuncture: clinical efficacy and mechanistic basis. Altern Ther Health Med. 2007:13(3):38-48.

27. StenerVictorin E, Waldenstrom U, Andersson SA, Wikland M. Reduction of blood flow impedance in the uterine arteries of infertile women with electro-acupuncture. Hum Reprod. 1996;11(6):1314-7. 
28. Balk J, Catov J, Horn B, Gecsi K, Wakim A. The relationship between perceived stress, acupuncture, and pregnancy rates among IVF patients: a pilot study. Complement Ther Clin Pract. 2010;16(3):154-7.

29. Domar AD, Meshay I, Kelliher J, Alper M, Powers RD. The impact of acupuncture on in vitro fertilization outcome. Fertil Steril. 2009;91(3):723-6.

30. Sniezek DP, Siddiqui IJ. Acupuncture for treating anxiety and depression in women: a clinical systematic review. Med Acupuncture. 2013;25(3):164-72.

31. El-Toukhy T, Sunkara SK, Khairy M, Dyer R, Khalaf Y, Coomarasamy A. A systematic review and meta-analysis of acupuncture in in vitro fertilisation. BJOG. 2008;115(10):1203-13.

32. Manheimer E, Zhang G, Udoff L, Haramati A, Langenberg P, Berman BM, Bouter LM. Effects of acupuncture on rates of pregnancy and live birth among women undergoing in vitro fertilisation: systematic review and meta-analysis. BMJ. 2008;336(7643):545-9.

33. Ng EH, So WS, Gao J, Wong YY, Ho PC. The role of acupuncture in the management of subfertility. Fertil Steril. 2008;90(1):1-13.

34. El-Toukhy T, Khalaf Y. The impact of acupuncture on assisted reproductive technology outcome. Curr Opin Obstet Gynecol. 2009;21(3):240-6.

35. Sunkara SK, Coomarasamy A, Khalaf Y, El-Toukhy T. Acupuncture and in vitro fertilization: updated meta-analysis. Hum Reprod. 2009;24(8):2047-8.

36. Qu F, Zhou J, Ren RX. Effects of acupuncture on the outcomes of in vitro fertilization: a systematic review and meta-analysis. J Altern Complement Med. 2012;18(5):429-39.

37. Zheng $\mathrm{CH}$, Huang GY, Zhang MM, Wang W. Effects of acupuncture on pregnancy rates in women undergoing in vitro fertilization: a systematic review and meta-analysis. Fertil Steril. 2012;97(3):599-611.

38. Zheng $\mathrm{CH}$, Zhang MM, Huang GY, Wang W. The role of acupuncture in assisted reproductive technology. Evidence-based complementary and alternative medicine : eCAM 2012; 2012. p. 543924.

39. Cheong YC, Dix S, Hung Yu Ng E, Ledger WL, Farquhar C. Acupuncture and assisted reproductive technology. The Cochrane database of systematic reviews, vol. 7; 2013. p. CD006920.

40. Manheimer E, van der Windt D, Cheng K, Stafford K, Liu J, Tierney J, Lao L, Berman BM, Langenberg P, Bouter LM. The effects of acupuncture on rates of clinical pregnancy among women undergoing in vitro fertilization: a systematic review and meta-analysis. Hum Reprod Update. 2013;19(6):696-713.

41. Shen C, Wu M, Shu D, Zhao X, Gao Y. The role of acupuncture in in vitro fertilization: a systematic review and meta-analysis. Gynecol Obstet Investig. 2014.

42. Qian Y, Xia XR, Ochin H, Huang C, Gao C, Gao L, Cui YG, Liu JY, Meng Y. Therapeutic effect of acupuncture on the outcomes of in vitro fertilization: a systematic review and meta-analysis. Arch Gynecol Obstet. 2017;295(3):543-58.

43. Jo J, Lee YJ. Effectiveness of acupuncture in women with polycystic ovarian syndrome undergoing in vitro fertilisation or intracytoplasmic sperm injection: a systematic review and meta-analysis. Acupunct Med. 2017;35(3):162-70.

44. Stener-Victorin E. Acupuncture in in vitro fertilisation: why do reviews produce contradictory results? Focus Altern Complement Ther. 2009;14:8-11.

45. White A. Western medical acupuncture: a definition. Acupunct Med. 2009; 27(1):33-5.

46. Higgins J, Altman D. Chapter 8: Assessing risk of bias in included studies. In: Higgins JPT, Green S, editors. Cochrane Handbook for Systematic Reviews of Interventions Version 510 [updated March 2011]. Available from http:// handbook-5-1.cochrane.org/.

47. Deeks J, Higgins J, Altman D. Chapter 9: analysing data and undertaking meta-analyses. In: JPT H, Green S, editors. Cochrane Handbook for Systematic Reviews of Interventions Version 510 [updated March 2011]. Available from http://handbook-5-1.cochrane.org/.

48. Nelson SM, Lawlor DA. Predicting live birth, preterm delivery, and low birth weight in infants born from in vitro fertilisation: a prospective study of 144,018 treatment cycles. PLoS Med. 2011;8(1):e1000386.

49. Bai L, Qin W, Tian J, Liu P, Li L, Chen P, Dai J, Craggs JG, von Deneen KM, Liu Y. Time-varied characteristics of acupuncture effects in $\mathrm{fMRI}$ studies. Hum Brain Mapp. 2009;30(11):3445-60.

50. Han JS. Acupuncture and endorphins. Neurosci Lett. 2004;361(1-3):258-61.

51. Thompson SG, Sharp SJ. Explaining heterogeneity in meta-analysis: a comparison of methods. Stat Med. 1999;18(20):2693-708.

52. Morgenstern $\mathrm{H}$. Uses of ecologic analysis in epidemiologic research. Am J Public Health. 1982;72(12):1336-44.

53. Greenland S. Quantitative methods in the review of epidemiologic literature. Epidemiol Rev. 1987;9:1-30.

54. Berlin JA, Santanna J, Schmid CH, Szczech LA, Feldman HI. Individual patient- versus group-level data meta-regressions for the investigation of treatment effect modifiers: ecological bias rears its ugly head. Stat Med. 2002;21(3):371-87.

55. Egger M, Davey Smith G, Schneider M, Minder C. Bias in meta-analysis detected by a simple, graphical test. BMJ. 1997;315(7109):629-34.

56. Smith CA, De Lacey SI, Chapman M, Ratcliffe J, Norman RJ, Johnson NP, Boothroyd C, Fahey P. Effect of acupuncture VS sham acupuncture on live births among women undergoing in vitro fertilization a randomized clinical trial. Jama. 2018;319(19):1990-8.

57. Morin SJ, Frattarelli JL, Franasiak JM, Juneau CR, Scott RT. Laser acupuncture before and after embryo transfer improves in vitro fertilization outcomes: a four-armed randomized controlled trial. Medical. 2017;29(2):56-65.

58. Gillerman K, Rehman N, Dilgil M, Homburg R. The impact of acupuncture on IVF success rates: a randomized controlled trial. Hum Reprod. 2016;31:i89.

59. Craig LB, Rubin LE, Peck JD, Anderson M, Marshall LA, Soules MR. Acupuncture performed before and after embryo transfer: a randomized controlled trial. J Reprod Med. 2014;59(5-6):313-20.

60. Rashidi $\mathrm{BH}$, Tehrani ES, Hamedani NA, Pirzadeh L. Effects of acupuncture on the outcome of in vitro fertilisation and intracytoplasmic sperm injection in women with polycystic ovarian syndrome. Acupunct Medicine. 2013;31(2):151-6.

61. Moy I, Milad MP, Barnes R, Confino E, Kazer RR, Zhang XQ. Randomized controlled trial: effects of acupuncture on pregnancy rates in women undergoing in vitro fertilization. Fertil Steril. 2011;95(2):583-7.

62. Feliciani E, Ferraretti AP, Paesano C, Pellizzaro E, Magli MC, Gianaroli L. The role of acupuncture in ART: preliminary results of an ongoing prospective randomized study. Hum Reprod. 2011;26:i230.

63. So EW, Ng EH, Wong YY, Yeung WS, Ho PC. Acupuncture for frozen-thawed embryo transfer cycles: a double-blind randomized controlled trial. Reprod BioMed Online. 2010;20(6):814-21.

64. Madaschi C, Braga DP, Figueira RC, laconelli A Jr, Borges E Jr. Effect of acupuncture on assisted reproduction treatment outcomes. Acupunct Med. 2010;28(4):180-4.

65. Andersen D, Lossl K, Nyboe Andersen A, Furbringer J, Bach H, Simonsen J, Larsen EC. Acupuncture on the day of embryo transfer: a randomized controlled trial of 635 patients. Reprod BioMed Online. 2010;21(3):366-72.

66. So E, Ng E, Wong Y, Lau E, Yeung W, Ho P. A randomized double blind comparison of real and placebo acupuncture in IVF treatment. Hum Reprod. 2009;24(2):341-8.

67. Smith C, Coyle M, Norman RJ. Influence of acupuncture stimulation on pregnancy rates for women undergoing embryo transfer. Fertil Steril. 2006; 85(5):1352-8.

68. Sator-Katzenschlager SM, Wolfler MM, Kozek-Langenecker SA, Sator K, Sator PG, Li BW, Heinze G, Sator MO. Auricular electro-acupuncture as an additional perioperative analgesic method during oocyte aspiration in IVF treatment. Hum Reprod. 2006;21(8):2114-20.

69. Humaidan P, Stener-Victorin E. Pain relief during oocyte retrieval with a new short duration electro-acupuncture technique-an alternative to conventional analgesic methods. Hum Reprod. 2004;19(6):1367-72.

70. Stener Victorin E, Waldenstrom U, Wikland M, Nilsson L, Hagglund L, Lundeberg $T$. Electro-acupuncture as a peroperative analgesic method and its effects on implantation rate and neuropeptide $Y$ concentrations in follicular fluid. Hum Reprod. 2003;18(7):1454-60.

71. Paulus WE, Zhang M, Strehler E, Seybold B, Sterzik K. Placebo-controlled trail of acupuncture effects in assisted reproduction therapy. Hum Reprod. 2003; 18:18-9.

72. Stener-Victorin $\mathrm{E}$, Waldenström U, Nilsson L, Wikland M, Janson PO. A prospective randomized study of electro-acupuncture versus alfentanil as anaesthesia during oocyte aspiration in in-vitro fertilization. Hum Reprod. 1999;14:2480-4

73. Yuan Y, Ren J, Huang L. Chinese English dictionary of traditional Chinese medicine. Beijing: People's medical publishing house; 1997.

74. Coughlan C, Ledger W, Wang Q, Liu F, Demirol A, Gurgan T, Cutting R, Ong $\mathrm{K}$, Sallam H, Li TC. Recurrent implantation failure: definition and management. Reprod BioMed Online. 2014;28(1):14-38.

75. Zeyneloglu HB, Onalan G. Remedies for recurrent implantation failure. Semin Reprod Med. 2014;32(4):297-305

76. Simon A, Laufer N. Repeated implantation failure: clinical approach. Fertil Steril. 2012;97(5):1039-43.

77. Urman B, Yakin K, Balaban B. Recurrent implantation failure in assisted reproduction: how to counsel and manage. A. General considerations and treatment options that may benefit the couple. Reprod BioMed Online. 2005;11(3):371-81. 
78. Matthiesen SM, Frederiksen Y, Ingerslev HJ, Zachariae R. Stress, distress and outcome of assisted reproductive technology (ART): a meta-analysis. Hum Reprod. 2011;26(10):2763-76.

79. Ebbesen SM, Zachariae R, Mehlsen MY, Thomsen D, Hojgaard A, Ottosen L, Petersen T, Ingerslev HJ. Stressful life events are associated with a poor invitro fertilization (IVF) outcome: a prospective study. Hum Reprod. 2009; 24(9):2173-82

80. Thiering P, Beaurepaire J, Jones M, Saunders D, Tennant C. Mood state as a predictor of treatment outcome after in vitro fertilization/embryo transfer technology (IVF/ET). J Psychosom Res. 1993;37(5):481-91.

81. Maughanand TA, Zhai XP. The acupuncture treatment of female infertility with particular reference to egg quality and endometrial receptiveness. J Chin Med. 2012(98):13-21.

82. Johansson J, Stener-Victorin E. Polycystic ovary syndrome: effect and mechanisms of acupuncture for ovulation induction. Evidence-based complementary and alternative medicine : eCAM, vol. 2013; 2013. p. 762615.

83. Johansson J, Redman L, Veldhuis PP, Sazonova A, Labrie F, Holm G, Johannsson G, Stener-Victorin E. Acupuncture for ovulation induction in polycystic ovary syndrome: a randomized controlled trial. Am J Phys Endocrinol Metab. 2013;304(9):E934-43.

84. Mo X, Li D, Pu Y, Xi G, Le X, Fu Z. Clinical studies on the mechanism for acupuncture stimulation of ovulation. J Tradit Chin Med. 1993;13(2):115-9.

85. Li J, Cui W, Sun W, Wen J. Effect of electroacupuncture on egg quality and tumor necrosis factor -a of patients with polycystic ovarian syndrome. World J Acupuncture - Moxibustion. 2014;24(3):9-15.

86. Cakmak YO. Electroacupuncture reduces uterine artery blood flow impedance. Taiwan J Obstet Gynecol. 2010;49(3):390 author reply 391.

87. Stener-Victorin E, Fujisawa S, Kurosawa M. Ovarian blood flow responses to electroacupuncture stimulation depend on estrous cycle and on site and frequency of stimulation in anesthetized rats. J Appl Physiol (1985). 2006; 101(1):84-91.

88. Smith CA, Ussher JM, Perz J, Carmady B, de Lacey S. The effect of acupuncture on psychosocial outcomes for women experiencing infertility: a pilot randomized controlled trial. J Altern Complement Med. 2011;17(10):923-30.

89. Sutton C, Pentland S, Roberts J. A comparison of stress levels in women undergoing single versus multiple acupuncture session prior to embryo transfer. Fertil Steril. 2015;103(2):e36-7.

90. Magarelli PC, Cridennda DK, Cohen M. Changes in serum cortisol and prolactin associated with acupuncture during controlled ovarian hyperstimulation in women undergoing in vitro fertilization-embryo transfer treatment. Fertil Steril. 2009;92(6):1870-9.

91. Zhang Y, Phy J, Orlando J, Garos S, Penrose L, Prien S, Huang JC. Effects of a delphi consensus acupuncture treatment protocol on the levels of stress and vascular tone in women undergoing in vitro fertilization: a randomized clinical pilot study preliminary findings. Global Advances in Health and Medicine. 2018;7:109-10.

92. Bhattacharya S, Maheshwari A, Mollison J. Factors associated with failed treatment: an analysis of 121,744 women embarking on their first IVF cycles. PLoS One. 2013;8(12):e82249.

93. Cetin MT, Kumtepe $Y$, Kiran H, Seydaoglu G. Factors affecting pregnancy in IVF: age and duration of embryo transfer. Reprod BioMed Online. 2010;20(3): 380-6.

94. Roberts SA, Hirst WM, Brison DR, Vail A. Embryo and uterine influences on IVF outcomes: an analysis of a UK multi-Centre cohort. Hum Reprod. 2010; 25(11):2792-802.

95. van Loendersloot LL, van Wely M, Limpens J, Bossuyt PM, Repping S, van der Veen F. Predictive factors in in vitro fertilization (IVF): a systematic review and meta-analysis. Hum Reprod Update. 2010;16(6):577-89.

96. Templeton A, Morris JK, Parslow W. Factors that affect outcome of in-vitro fertilisation treatment. Lancet. 1996;348(9039):1402-6.

97. Hrobjartsson A, Gotzsche PC: Placebo treatment versus no treatment. The Cochrane database of systematic reviews 2003(1):Cd003974.

98. Hrobjartsson A, Gotzsche PC. Is the placebo powerless? Update of a systematic review with 52 new randomized trials comparing placebo with no treatment. J Intern Med. 2004;256(2):91-100.

99. Hrobjartsson A, Gotzsche PC: Placebo interventions for all clinical conditions. The Cochrane database of systematic reviews 2010(1):Cd003974.

100. Wood L, Egger M, Gluud LL, Schulz KF, Juni P, Altman DG, Gluud C, Martin RM, Wood AJ, Sterne JA. Empirical evidence of bias in treatment effect estimates in controlled trials with different interventions and outcomes: meta-epidemiological study. BMJ. 2008;336(7644):601-5.
101. Lund I, Naslund J, Lundeberg T. Minimal acupuncture is not a valid placebo control in randomised controlled trials of acupuncture: a physiologist's perspective. Chin Med. 2009;4(1).

102. Lundeberg T, Lund I, Sing A, Naslund J. Is placebo acupuncture what it is intended to be? Evidence-based complementary and alternative medicine : eCAM, vol. 2011; 2011. p. 932407

103. Kaptchuk TJ, Stason WB, Davis RB, Legedza AR, Schnyer RN, Kerr CE, Stone DA, Nam BH, Kirsch I, Goldman RH. Sham device v inert pill: randomised controlled trial of two placebo treatments. BMJ. 2006;332(7538):391-7.

104. Meissner K, Distel H, Mitzdorf U. Evidence for placebo effects on physical but not on biochemical outcome parameters: a review of clinical trials. BMC Med. 2007:5:3.

105. Sterne JA, Gavaghan D, Egger M. Publication and related bias in metaanalysis: power of statistical tests and prevalence in the literature. J Clin Epidemiol. 2000;53(11):1119-29.

106. Sedgwick P. What is publication bias in a meta-analysis? BMJ. 2015;351: h4419.

107. Sterne J, Egger M, Moher D. Chapter 10: addressing reporting biases. In: JPT $\mathrm{H}$, Green S, editors. Cochrane Handbook for Systematic Reviews of Interventions Version 510 [updated March 2011]. Available from http:// handbook-5-1.cochrane.org/.

108. Glasziou PP, Irwig LM. An evidence based approach to individualising treatment. BMJ. 1995;311(7016):1356-9.

109. Smith GD, Egger M. Who benefits from medical interventions? Br Med J. 1994;308(6921):72-4.

110. Stuck AE, Rubenstein LZ, Wieland D. Bias in meta-analysis detected by a simple, graphical test. Asymmetry detected in funnel plot was probably due to true heterogeneity. BMJ. 1998;316(7129):469 author reply 470-461.

111. Cui W, Liu LL, Sun W, Kong W. Effects of electroacupuncture on in vitro fertilization and embryo transplantation in the patient of infertility with different syndromes. Zhongguo Zhen Jiu. 2008;28(4):254-6.

\section{Ready to submit your research? Choose BMC and benefit from:}

- fast, convenient online submission

- thorough peer review by experienced researchers in your field

- rapid publication on acceptance

- support for research data, including large and complex data types

- gold Open Access which fosters wider collaboration and increased citations

- maximum visibility for your research: over $100 \mathrm{M}$ website views per year

At $\mathrm{BMC}$, research is always in progress.

Learn more biomedcentral.com/submissions 